\title{
Circadian Rhythms of Physiological Functions of the Pregnant Woman and the Fetus and the Biological Solemnity Thereof
}

\author{
Medea G. Zarnadze \\ K.V. Chachane Institute of Perinatology, Obstetrics and Gynecology, Tbilisi, Republic of Georgia \\ Email: mgz13@rambler.ru
}

Received 18 April 2016; accepted 24 July 2016; published 27 July 2016

Copyright (c) 2016 by author and Scientific Research Publishing Inc.

This work is licensed under the Creative Commons Attribution International License (CC BY). http://creativecommons.org/licenses/by/4.0/

(c) (i) Open Access

\section{Abstract}

The basic rhythms of nature that left their imprint on the existence of all living organism on the Earth, arose under the influence of the Earth's rotation relative to the Sun, the Moon and other planets and stars of the Universe. This periodicity gave rise to the rhythm that has become essential for their life. Life is a continual chemical process of building up and breaking down of organic substances, which results from the substance exchange between an organism and the environment. This makes it impossible for a living organism to exist without the external environment. Since 1978-1979 we have been carrying out a task-oriented research with the aim to approximate the moment when we are able to answer all these questions. Daily fluctuations of cardiac and motor activity of the fetus have been studied (uninterrupted daily recording of fetal ECG); polysomnography of nocturnal sleep was recorded; daily fluctuations of endocrine system activity in the pregnant were studied. A correlation was made between the functional state of maternal sleepwakefulness biological rhythm, biological clock of the human fetus and the "light-darkness" cycle of a 24-hour solar day. In the process of the study we have developed an original method of day-to-day analysis of maternal and fetal ECGs. It has been established that a healthy fetus has distinct, diurnal variations of physiological functions. The fetal biorhythms coordinate with the status of the maternal organism being, however, in an opposite phase. The curve of the dynamics of fetal physiological system functioning shows a biphasic nature (one-phase in adults). "Active" and "quiet" (sleep-like) periods have been singled out in the human fetus. No reaction is observed in "quiet" periods. However, the "zero"-type fetal reaction recorded by us within the period from 2 p.m. to 9 p.m. does not indicate unsatisfactory condition of the fetus but rather is suggestive of a definite reduction of functional levels of the fetal physiological systems, which is necessary for vital activity. Although conventionally recognized as an indicator of poor state of the fetus, this type only calls for precise attention when recorded in fetal "active" hours. The present study has been the first in the world's medicine and biorhythmology to detect and establish the daily rhythms of 
cardiac and motor activity in the human fetus.

Keywords

Circadian Rhythms, Biorhythms, Pregnant Woman, Human Fetus, Puls, Beats Per Minute, Cardio Tachogram, Abdominal Electrocardiogram, Lokomotor Activity, Test, “Activ”, “Quiet”, Stars, Universe, Space

\section{Introduction}

Selection of the criterion for dynamic monitoring of the fetus condition during pregnancy to control the efficiency of the study conducted, as well as for the comparison of the intensity of intrauterine suffering of the fetus with various types of obstetrical and extragenital pathology is of utmost importance in obstetrics.

Identification of the mechanism of adaptation of the fetal organism to the factors of the environment and the mechanisms, serving as the basis for the compensation of disordered functions, remain to be the key problem in perinatal medicine [1]-[5]. Inasmuch as the influence of the environment on the fetus is formed by an uninterrupted impact of physical, chemical, social and other factors, the counter-response of organism, in its turn, lies in the similarly uninterrupted fluctuations of bio-synthetic processes, corresponding to these impacts [6]-[10].

In this regard, studies, aiming at the investigation of the diurnal rhythm of various physiologic functions both in the body of the mother and the fetus are of particular interest [11]-[14]. The aim of our study (1978-1979 y.) is: "The interdependence of functional systems of a pregnant woman and the fetus". An immediate and most efficient access to the study of daily rhythms of the fetus is the monitoring and analysis of fetal heart rate and the motor activity. The frequency of fetal heartbeat is twice as high as maternal heartbeat, which permits to conclude that the fetal heartbeat rhythm is a unique, autonomic system, independent of the maternal rhythm. The fetal heartbeat can be seen and heard as early as within $4-4.5$ weeks of pregnancy. And, if we take into account the velocity of cell reproduction from the moment of conception, it becomes clear that the PULSE of fetal development has already been formed from the very first instants of the conception. This is the substantiation of the goal of our study.

\section{Material and Method}

\section{Contents}

Main objectives of study:

1) Identification of daily rhythms of cardiac and motion activity of the fetus during physiological pregnancy.

2) Study and identification of interrelation of bio-rhythms of the mother and the fetus.

3) Study of characteristics of the fetal response to the functional load taking the bio-rhythms into consideration.

4) Definition of the diagnostic value of various functional probes upon isolated and combined application.

Material and methods of study N1 (tasks N1 and N2).

In view of the identification of the daily rhythm of cardiac and motion activity of the fetus during pregnancy, as well as for the evaluation of the functional condition, the method of external ECG of the fetus was applied, which allowed to simultaneously obtain the findings regarding the alteration of maternal and fetal heartbeat frequency during 24 hours. The ECGs were recorded by two electrodes adjusted on the median line of the front abdominal wall of the pregnant at the fundus and above the symphisis. Electrodes were fixed by means of special rubber belt or glue. Routine conditions of life and regime for the pregnant woman have been strictly maintained during the study of daily rhythms of cardiac and motion activity of the mother and the fetus. The test was recorded in a shielded room following 2 - 3 days of uninterrupted preliminary adaptation during 24 hours with intermittent short breaks (meals, reading and listening to favorite tunes and songs according to the preferences of the woman, cleaning, 10-minute walks four times per 24 hours, periodic contact with relatives; the pregnant woman was as well allowed to sit or lie in any comfortable position). ECGs were recorded almost continuously during 24 hours with no night sleep interruption. Every manipulation in the course of the study was agreed with the pregnant and their relatives. In our clinic, all pregnant women gave birth to healthy children with $9-10$ points according to Apgar scale. All newborns and the mothers remained in the ward during 9 days. (Those 
years, pregnant women with children stayed in the hospital during 9 days upon physiological delivery and 12 days upon pathologic cases. Pregnancy and delivery were free of charge).

The recorded material was processed as to calculate FHR of the mother and the fetus within five-second intervals and to transfer the data to a cardiotachogram, which was the diagram of internal increment fluctuations of FHR of the pregnant woman and the fetus. 24 cardiograms were recorded by hour from 1 am to 2 am, from 2 am to 3 am, from 3 am to 4 am etc. of the next day.

The analysis of the hourly cardiograms was held according to the following parameters: identification of hourly fluctuations of FHR of the mother of the fetus, internal increment fluctuations of FHR, basal rhythm. Movements of the fetus were registered upon fluctuations of isoelectric line of external ECG of the fetus, calculated according hours and concluded into the diagram of hourly movements of the fetus. Studying the daily rhythms of the mother and the fetus, we have examined 18 volunteer pregnant women aged 19 to 26 years at the gestational period of 36 to 40 weeks of physiologic pregnancy (in all cases, the fetus was in the cephalic presentation). The occupation, life conditions and the 24-hour regime of the women, were taken into account, and their heredity was considered. Healthy women without disorders of basic of sleep-wakefulness bio-rhythm have been selected.

Material and methods of study N2 (tasks N3 and N4).

Simultaneously with the studies, we have daily held the non-recurrent records of ECG to detect the functional condition of the fetus [the hospital was equipped with the Diagnostic Center and the Department of Pathology]. All pregnant women admitted to these departments were mandatorily subject to external ECG recordings of the fetus. ECG of the fetus was registered during 20 - 25 minutes. 10 minutes after the onset of the background recordings, the functional probes were done using: breath-holding on exhalation, exercise stress, audio stimulus, and non-stress test [NST]. The pregnant held the breath according to own capacities. With sound stimulation to the anterior abdominal wall, a telephone was placed to the projection of the fetal head to transmit a constant sound with the duration of 60 seconds and the frequency within the range of $500-1000 \mathrm{~Hz}$. The pregnant woman was offered to put on headphones to exclude the impact of the audio stimulus on the mother's organism. Exercise stress was conducted as bending and unbending of the trunk, 10 times during 30 - 35 seconds. NST registered the changes in the heart rate frequency (FHR) in response to the motion activity of the fetus.

To reveal the most informative functional test, we compared their features at isolated and combined application. In isolated application of the functional load, one of the above mentioned tests was used. In combined application, two or three control tests were used in the dynamics of one record for the identification of intrauterine condition of the fetus. The reaction of the fetus to NST was registered both in isolated in combined testing. The condition of the fetus with isolated application of a physical load was evaluated by the reaction of the fetus to the test. The condition of the fetus in combined application of the control tests was evaluated by the reaction of the fetus to a physical load, that in isolated application had the highest diagnostic value. Studies were conducted using the calculation of two indices: the index of fetal reactivity to the test and the diagnostic value of the test.

The index of reactivity was defined by the quantity of fetal reactions to the test according to the following formula: $\frac{(x) \times 100 \%}{n}$ where " $x$ " is the totality of fetal reactions to the test, followed by the alteration of FHR (fetal heart rate) and " $n$ " is the number of fetuses under study, whose condition was identified using one type of the mentioned loads. Diagnostic value of the test was determined according to the conclusion data, obtained on the basis of the studies of external fetal ECGs using the assessment by Apgar scale and the evaluation of the course of early neo-natal period which was calculated according to the following formula: $\frac{(y) \times 100 \%}{n}$ where " $y$ " is the number of fetuses, whose condition was precisely estimated during the ECG recording.

The functional condition of the fetus was estimated according to the data of the cardiotachogram (CTG) analysis with the three segments being singled out for a separate analysis: background CTG, functional stress and CTG of post-functional stress.

The background CTG was to show the data on FHR fluctuations, the basal rhythm, internal increment fluctuations. The CTG segment-functional load-was to emphasize the character of changes in FHR of the fetus resulting from the tests, latent period, time of FHR restoration to the basal value. The CTG segment was to determine the level of the basal rhythm and FHR fluctuations after the load.

To study the characteristics of fetal reaction to functional tests, related to the daily rhythms of the heart and motion activity, external ECGs of the fetus was registered in various periods of the day. The time of ECG re- 
cording of was strictly accounted. Differences of all indices were considered reliable at R 0.05 . We have studied 2407 fetuses for the identification of the fetus condition during pregnancy at the gestational term of $30-42$ weeks. Women with multi-fetal pregnancy were not included into the studies (CTG fails to allow simultaneous recording of two or more fetuses). 94 of 2407 pregnant volunteer women gave their consent on fetal ECG recording during the evening and night hours. Functional condition of the fetus was examined in dynamics with the interval of 8 days. They were classified into four groups. Examination of the fetuses of I, II and III groups were performed during hours "active" for the fetus (from $0900 \mathrm{~h}$ to $1400 \mathrm{~h}$ and from $1900 \mathrm{~h}$ to $0400 \mathrm{~h}$ ). Tests in group IV were performed during hours "passive" for the fetus (from $1400 \mathrm{~h}$ to $1900 \mathrm{~h}$ and from $0400 \mathrm{~h}$ to 0900 h). Groups I, II and III were sub-classified into two sub-groups. The sub-group A was composed of the fetuses of the mothers with physiologic pregnancy, the sub-group B was composed of the fetuses of the mothers with pathologic pregnancy [Hydrops gravidarum, Preeclampsia, Eclampsia, Hypertonia gravidarum, premature birth, Rh-negative pregnancy, hydramnion, hypamnion, burdened obstetrical history, anemia of pregnant women, diabetes mellitus etc.]. Group I was composed of 800 fetuses (the sub-group A-300, sub-group B—500), which were subject to all types of stresses plus NST for the identification of their condition. Group II was also composed of 800 fetuses (the sub-group A-300, sub-group B-500). In this group one of the tests with the addition of NST was used [isolated application of the test]. Group III was composed of 800 fetuses (the sub-group A400, sub-group B-400), which were subject to two or three tests without NST in dynamics [combination of tests]. Group IV was composed of 169 fetuses [sub-group A], that, when examined during "active” hours, expressed an "acceleration" type of reaction. In this group, sound stimulus and exercise stress were applied in combination in capacity of the loads.

The data of fetal ECG was compared according to Apgar scale and in respect of the neonatal period/the birth time. For greater reliability, the data obtained, were processed using Student's method of variable statistics Table 1.

Table 1. Hourly layout for 24-hour period frequencies of maternal and fetal heart rate rhythms ( 36 - 40 weeks gestation).

\begin{tabular}{|c|c|c|}
\hline Time of day (h) & Mother (bpm) & Fetus (bpm) \\
\hline $7-8$ & $66.5 \pm 1.3$ & $133 \pm 1.9$ \\
\hline $8-9$ & $68 \pm 1.2$ & $133 \pm 2.1$ \\
\hline $9-10$ & $68.5 \pm 1.3$ & $132 \pm 2.0$ \\
\hline $10-11$ & $72 \pm 1.4$ 楼 & $136 \pm 2.1$ \\
\hline $11-12$ & $76 \pm 1.4$ 畨 & $135 \pm 2.3$ \\
\hline $12-13$ & $79 \pm 1.3$ & $136 \pm 2.3$ \\
\hline $13-14$ & $79 \pm 1.2$ & $134 \pm 1.9$ \\
\hline $14-15$ & $75 \pm 1.2$ & $136 \pm 2.1$ \\
\hline $15-16$ & $76 \pm 1.1$ & $133 \pm 2.3$ \\
\hline $16-17$ & $76 \pm 1.1$ & $132 \pm 1.8$ \\
\hline $17-18$ & $76.5 \pm 1.2$ & $133 \pm 2.2$ \\
\hline $18-19$ & $79 \pm 1.0$ & $135 \pm 1.8$ \\
\hline $19-20$ & $71 \pm 1.1$ & $131 \pm 1.8$ \\
\hline $20-21$ & $72 \pm 1.1$ 畨 & $133 \pm 2.0$ \\
\hline $21-22$ & $69 \pm 1.2$ & $135 \pm 1.9$ 米 \\
\hline $22-23$ & $70 \pm 1.1$ & $134 \pm 1.8$ \\
\hline $23-24$ & $65 \pm 1.0$ & $136.5 \pm 2.0$ \\
\hline $24-1$ & $64 \pm 1.2$ & $138.5 \pm 1.9$ \\
\hline $1-2$ & $65 \pm 1.1$ & $138.5 \pm 2.2$ \\
\hline $2-3$ & $61 \pm 1.2$ & $133.5 \pm 1.9$ 米 \\
\hline $3-4$ & $64 \pm 1.1$ & $136 \pm 1.8$ \\
\hline $4-5$ & $62 \pm 1.1$ & $132 \pm 1.9$ \\
\hline $5-6$ & $66.5 \pm 1.1$ & $133 \pm 2.1$ \\
\hline $6-7$ & $68 \pm 1.0$ & $134 \pm 2.2$ \\
\hline
\end{tabular}

$\mathrm{p}<0.001$ (compared to heart rate at $7 \mathrm{am}$ ). $\mathrm{p}>0.05$. 


\section{Result and Discussion.}

\subsection{Interdependency and Concordance of Daily Rhythms of the Human Fetus with Bio-Rhythms of a Pregnant Woman}

Study of biologic rhythms is comprehensive if held uninterruptedly and the basic methodological sign of the analysis of biorhythms is the diversity of plans-simultaneous study of sundry indices, characterizing the amplitude, period and the phase of fluctuations. We performed uninterrupted, simultaneous recordings of two indices of the fetus: frequency of heart rate (FHR) and motion activity as well as heart beats (ECG) of the pregnant woman under the conditions that can be considered as quite physiological.

The analysis of the study on daily periodicity of the heart rhythm of healthy pregnant women at 07:00, 12:00, 17:00, 22:00, 02:00 o'clock revealed that $\mathrm{HR}$ at 07 am before taking food and fluids as well as before exercise stress corresponds to 66.5 beats $/ \mathrm{min}$. The following time point of interest is midday $-12 \mathrm{pm}$. At this time, HR was at the optimal point-79, 5 beats/min. In post-midday period -5 pm-FHR was 76,5 beats/min, at 10 $\mathrm{pm}-70,5$ beats/min and at 2 am 61 beats/min. The results evidenced the fluctuations of HR during 24 hours with the maximal level in daytime hours and with decrease during the night. The hourly analysis of fluctuations also revealed the increase of HR during the day. Statistic processing of the HR indices in a pregnant woman during the day and night time showed a reliable result $(\mathrm{p}<0.001)$.

The daily fluctuations of FHR revealed that at 7 a.m., FHR made 10 p.m. $133 \pm 1.9$ beats $/ \mathrm{min}$, at 12 p.m. $-136 \pm 2.3$ beats $/ \mathrm{min}$, at $1700 \mathrm{~h}-133 \pm 2.2$ beats $/ \mathrm{min}$, at $-134 \pm 1.7$ beats $/ \mathrm{min}$ and at 2 a.m. $-133 \pm 1.9$ beats/min. Statistic processing of the FHR indices of during 24 hours did not give a significant difference between the day and night values ( $p>0.5$ ). The hourly analysis of FHR also failed to reveal a certain increase during the daytime. As we can see from the Table 1, increase of heart beat frequency of the fetus up to $138 \pm$ 1.9 beats/min is observed during night hours from 12 a.m. to 2 a.m. and certifies statistically reliable $(\mathrm{p}<0.001)$ The increase FHR during this period of night in regard with the initial point, lower than the level at 7 a.m. (133 \pm 1.9 beats/min), whereas FHR of the mother is drastically decreased during the night time and increased in morning hours (Figure 1, Figure 2) he results obtained served as the ground for considerations.

Our data coincide with the results of the study by T.H. Hallbrugge [15] [16], indicating to the typical 24-hour rhythm in pregnant women. At the same time, FHR of the fetus is more or less equal during the day and the night and averagely constitutes $133.0 \pm 5.0$ beats $/ \mathrm{min}$.

T. Hoppenbrouere et al. [17] have defined that FHR of the fetus is not drastically altered upon vigilance and sleep of the mother, there is no link between the sleep phases of the mother and alterability of FHR of the fetus.

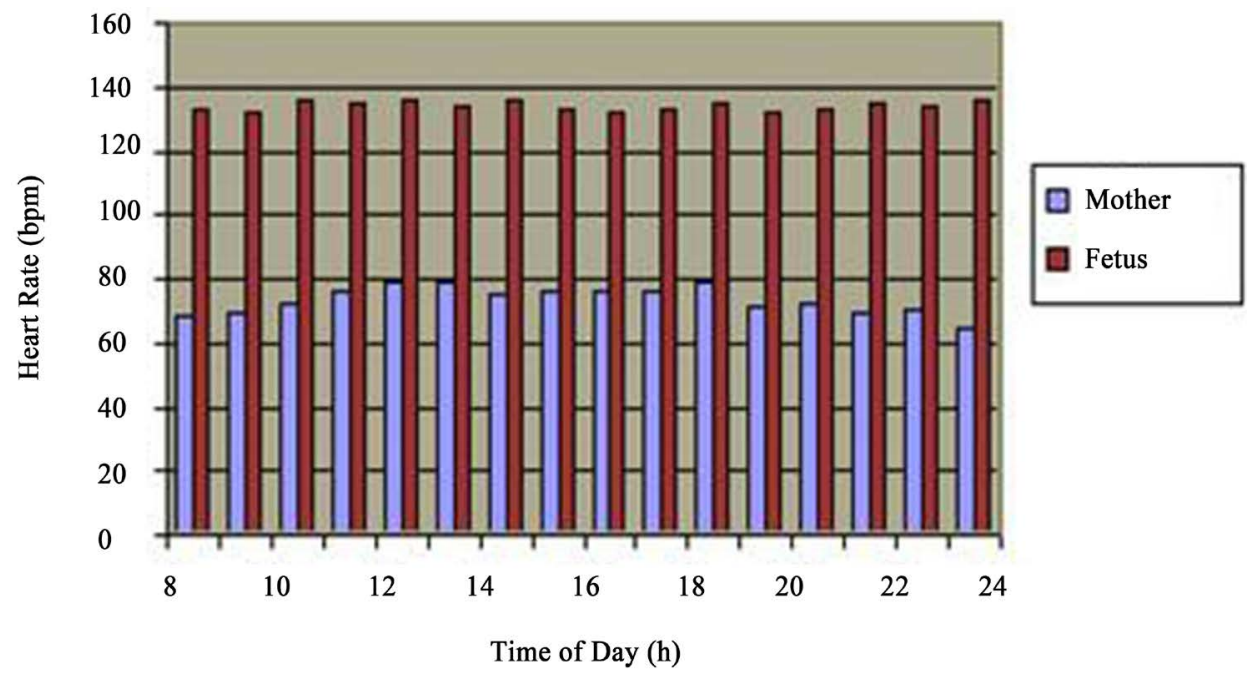

Figure 1. Daytime types of cardio tachograms. Hourly layout for 24-hour period frequencies of maternal and fetal heart rate rhythms (36 - 40 weeks gestation). clearly reveal the heartbeat biorhythm of a healthy pregnant woman showing the decrease in HRF at night hours and increase during the daytime (blue column); analysis of the alteration of frequency of heart rate of the fetus during 24 hours does not give the basis to discuss daily rhythms of heart rates of the fetus (vinous column). 


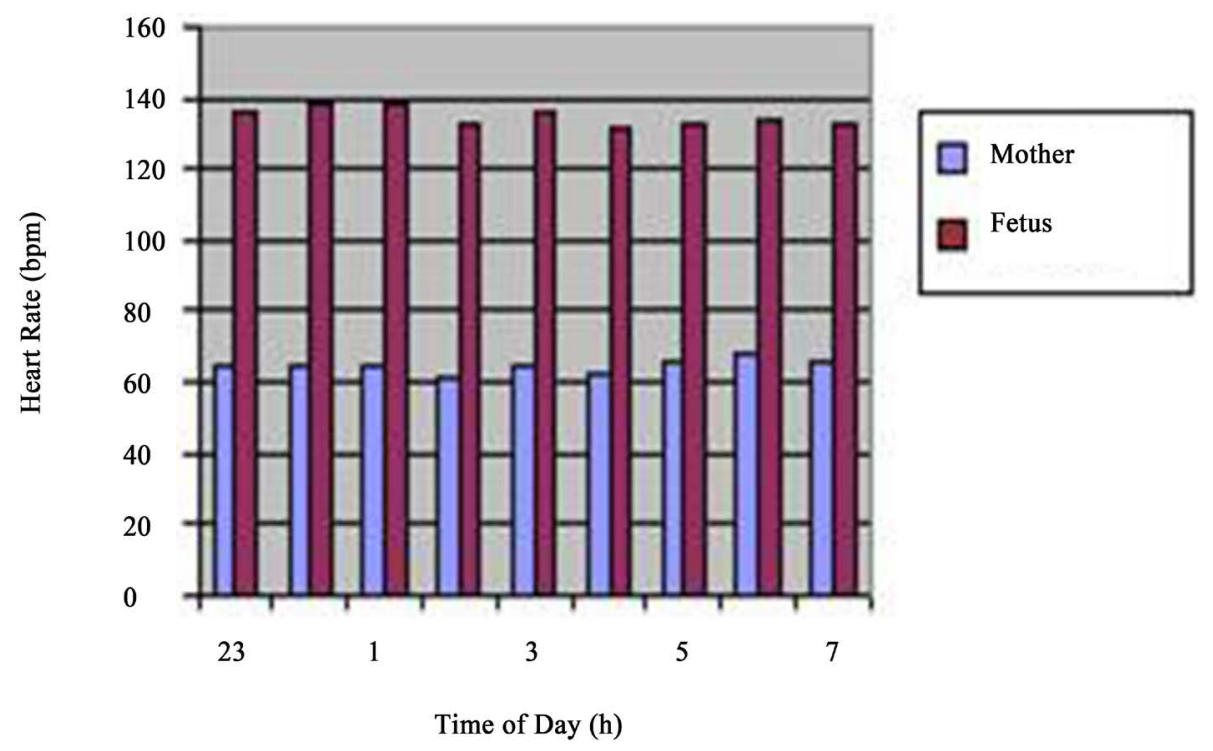

Figure 2. Night. hourly layout for 24-hour period frequencies of maternal and fetal heart rate rhythms (36 - 40 weeks gestation). clearly reveal the heartbeat biorhythm of a healthy pregnant woman showing the decrease in HRF at night hours and increase during the daytime (blue column); analysis of the alteration of frequency of heart rate of the fetus during 24 hours does not give the basis to discuss daily rhythms of heart rates of the fetus (vinous column).

A.M. Rudolph and M.A. Heymann, in their chronic experiments on lambs, have observed alterations of FHR, blood pressure and blood regurgitating from the right ventricle of heart in view of respiratory activity of the fetus. Deep, prolonged inhalation caused the increase of these parameters. Cardiac output of the fetus was altering almost exclusively at the expense of FHR. The authors discovered that the cardiac output of a mature animal with the increase of HR exceeding $160-180$ beats/min, is reduced and cardiac output of the fetus does not change even upon FHR of $300-325$ beats/min. Studies of N.A. Arkhangelskaya [18], held in experiment revealed feebly marked daily rhythm of temperature of the body of the fetus according to the configuration, reverse to the rhythm of the mother. The author attributes it to the formation of the conditioned reflex of thermoregulation, related to the decrease of maternal body temperature during the nighttime. N.N. Konstantinova [19], maintains that not only dependence of FHR of the fetus on sleep and wakefulness of the mother was established but also alterations of these indices during falling asleep and awakening of a pregnant woman were revealed.

Having put the task into agenda to study the laws of concordance of biorhythms of the mother and the fetus, we exposed the daily ECG of the fetus to the visual analysis and attached our attention to the heterogeneity of their structure, that enabled us to classify the four types of oscillation, registered on the hourly ECG in various combinations and sequence, continuously replacing one another. Each of the types of oscillation, different in form, amplitude and duration, was respectively named as: "pointed", "rounded", "flat" and "mixed" types of oscillation.

Type I-“pointed"-is frequent fluctuations of FHR during 5 - 10 seconds with intra-minute fluctuations of \pm 12 - 30 beats/min.

Type II- “rounded"-is represented by slow acceleration of FHR of the fetus to \pm 18 - 34 beats/min and slow deceleration to the initial index.

Type III- “flat"-is represented by small intra-minute fluctuations of \pm 1 - 5 beats $/ \mathrm{min}$.

Type IV_-“mixed”-is represented by basal rhythm of 120 - 150 beats/min and intra-minute fluctuations of \pm 7 - 15 beats/min (Figure 3).

Having dismembered the daily, hourly CTG (24 tachograms were made up for each pregnant woman), exposing it to the thorough visual and digital analysis, we clearly documented the irregularity of distribution of one or another type of oscillation on the daily rhythmogram. Calculating the quantity according to the period of time during which each type of CTG repeats within one hour of study, we paid attention to the fact that the period when the background CTG of the fetus expressed by "flat" (III) type of oscillation (52\% of registration time) 


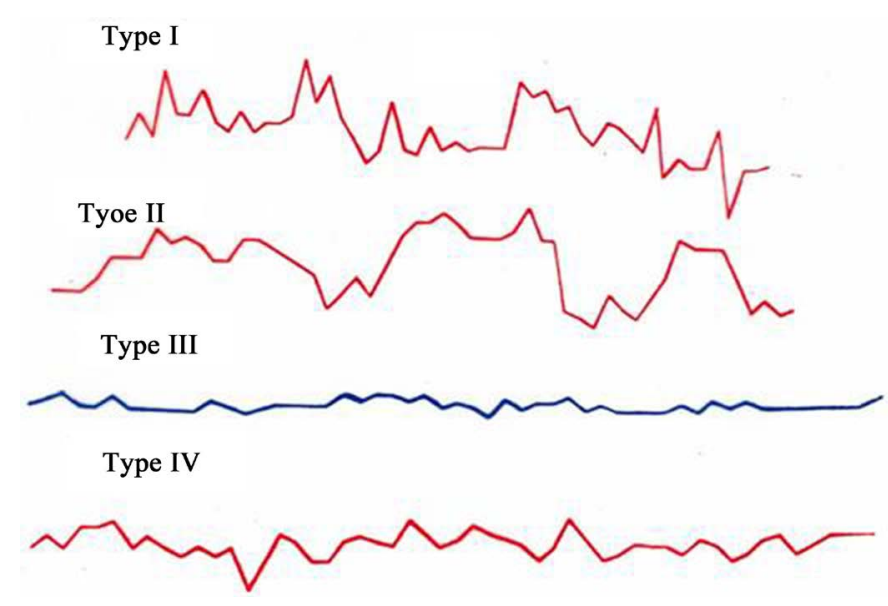

Figure 3. Types of fetus cardio taghograma. Type 1—“pointed”; Type 2-“rounded"; Type 3-“flat”; Type 4-“mixed”.

can be observed twice in 24 hrs with the prevalence over other types (I, II, IV) of oscillation. This period of time-from 4 a.m. to 9 a.m. (Figure 4). And from 2 p.m. to 7 p.m. (Figure 5), is called "passive" hours. During the rest of the time-from 9 a.m. to 2 p.m. (Figure 6), and from 7 p.m. to 4 a.m. (Figure 7) - the background CTG of the fetus was expressed by a "mixed" type of oscillation, against the background of which type I and type II oscillation were recorded (87.8\% of registration time). These periods of time are called "active" hours. tudies revealed that the concentration of type I oscillations in "passive" hours is four times whereas that of types II and IV is two times less than in "active" hours. Statistic processing of the indices of duration of the types of oscillation in "active" and "passive" hours revealed reliable results ( $\mathrm{p}<0.001)$.

Against the background of the "flat" (III) or "mixed" (IV) types of oscillation with various intensity, depending on the period of time, we can reveal the "pointed" (I) and "rounded" (II) types of oscillation. As we can see from the Tables 2-4, "mixed" (IV) type of oscillation during "active" hours constitutes $55.8 \%$ of total time of registration (316 min. $50 \mathrm{sec}$. out of $569 \mathrm{~min}$. of total time). "Rounded" (II) type of oscillation is registered during 165 minutes, which corresponds to 28.8\%, and "pointed" (I) type of oscillation is registered during 19 min. 5 sec. (3.3\%). "Flat" (III) type of oscillation within "active" hours is registered during $12.2 \%$ of registration time (Figure 8, Fetal Cardio tachogram "active" and "quiet” hours).

Within "passive" hours, the "flat" (III) type of oscillation is registered during 246 minutes of registration, which corresponds to $52 \%$ of total time. "Mixed" (IV) of oscillation, prevailing within "active" hours, is registered within "passive" hours during 136 minutes, which constitutes $28.7 \%$ of registration time.

"Pointed" (I) type of oscillation, within "passive" hours, is registered during 4 minutes, which is nine times rarer than in "active" hours. "Rounded" (II) type of oscillation is registered during 87 minutes or $18.5 \%$ of registration time. Statistical processing of the indices of duration of types of oscillation (I, II, III, IV) in "active" and "passive" hours of the fetus revealed the reliable results $(\mathrm{p}<0.001)$.

Registered daily ECG of a pregnant woman also was subject to analysis: CTG held account of registration time of a "flat" type of oscillation according to hours during the day. The data that we obtained, are provided in the Table 5, where you can see that from $23 \mathrm{~h} 00$ to $08 \mathrm{~h} 00$, concentration of a "flat" (III) type of oscillation on the hourly CTG is drastically increased compared to other periods of the day. Time from 23h00 to 08h00 coincides with the period of sleep of a pregnant woman, that is-with "passive" hours. Concentration of the "flat" (III) type of oscillation reaches the maximal point at $04 \mathrm{~h} 00-05 \mathrm{~h} 00-33$ minutes. During the sleep of a pregnant woman it fluctuates within 6 minutes (24h00 - 01h00) to 19 minutes (02h00 - 03h00). Since 08h00, concentration of the III type of oscillation drastically reduces (from $08 \mathrm{~h} 00$ to $09 \mathrm{~h} 00$ - constitutes 2 minutes) and during the day, upon vigilance of a pregnant woman, or is absent (from 11h00 to 13h00), or is registered on the hourly CTG during the short period of time, not exceeding 4 minutes.

The (Figure 9) provides graphic distribution of "active" and "passive" hours of a woman and intrauterine fetus upon physiological pregnancy.

Researches have revealed that concentration of the I type of oscillation within "passive" hours is four times, and of the and the III types, two times less than in "active" hours. The statistical processing of indices of duration 


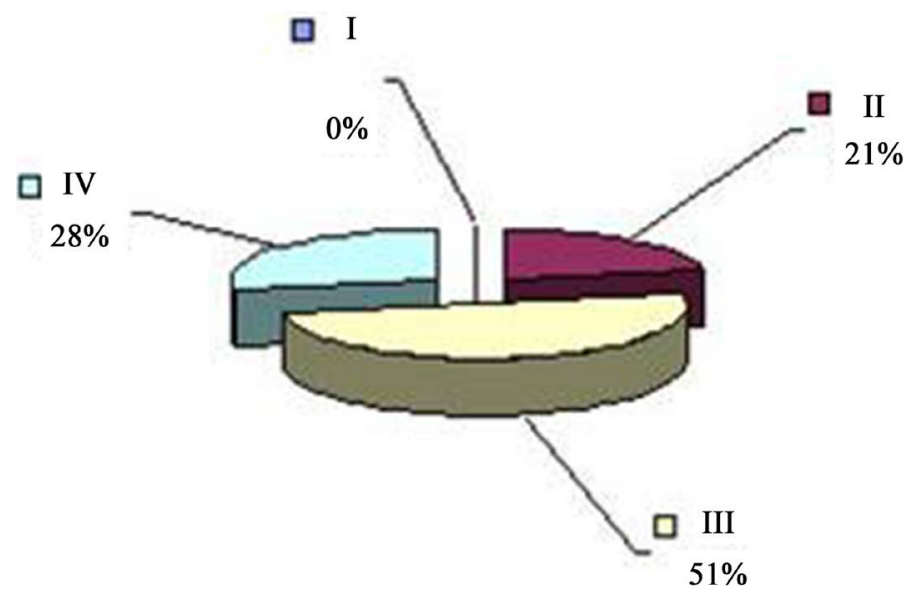

Figure 4. ECG of the fetus in "quiet” hours 51\% (4 a.m. to 9 a.m.) 36 - 40 weeks gestation ("quiet" hours-yellow; type III- “flat”; "active” hours-blue, type I-“pointed”; bordeaux, type II-“rounded” and green, type IV—_mixed”).

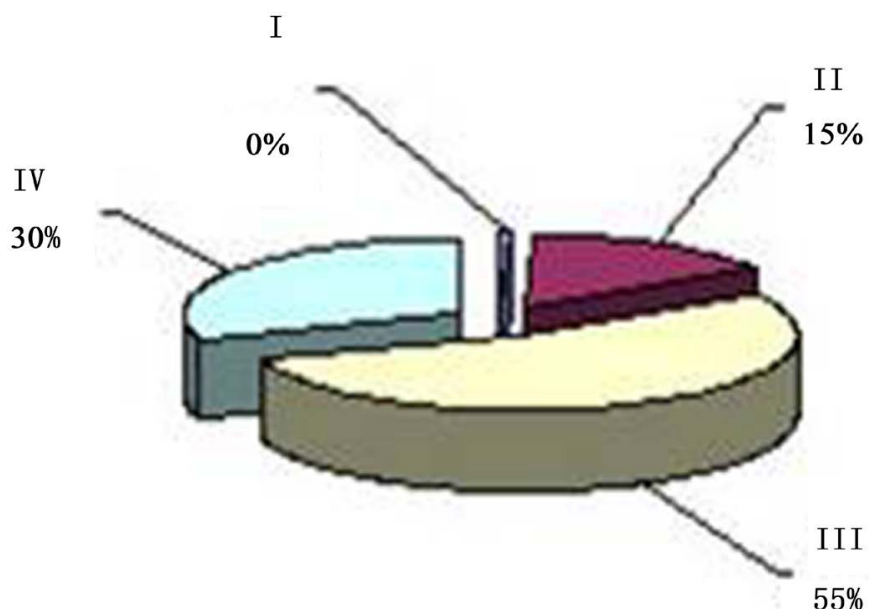

Figure 5. ECG of the fetus in "quiet” hours 55\% (2 p.m. to 7 p.m.) 36 - 40 weeks gestation ("quiet" hours-yellow; type III- "flat"; "active” hours-blue, type I_-"pointed”; bordeaux, type II—_rounded” and green, type IV—_mixed”).

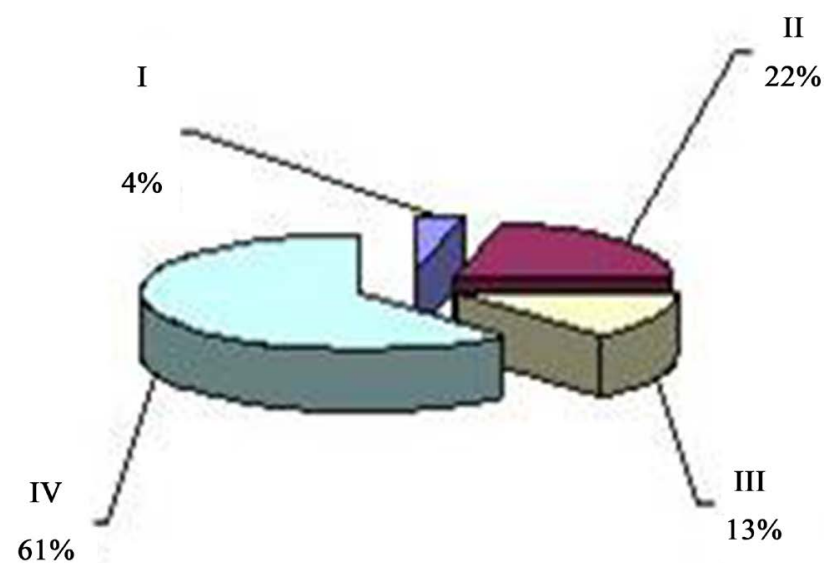

Figure 6. ECG activity of the fetus in $87 \%$ "active” hours (9 a.m. to 2 p.m.) 36 40 weeks gestation ("active” hours-blue, type I- “pointed”; bordeaux, type II"rounded” and green, type IV_—“mixed”; “quiet” hours—yellow; type III— “flat”). 


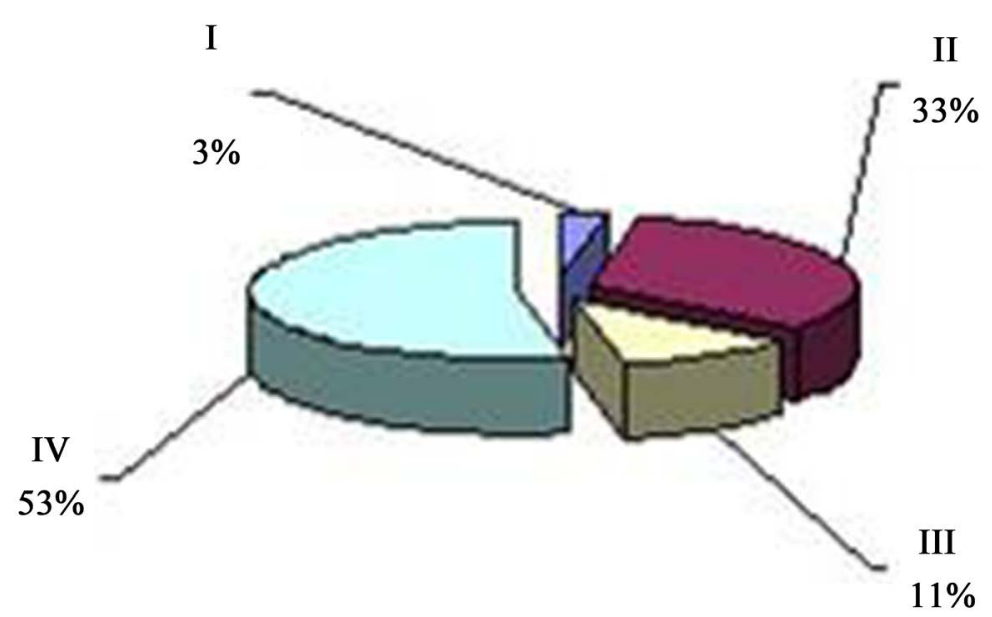

Figure 7. ECG activity of the fetus in $89 \%$ "active” hours (from 7 p.m. to 4 a.m.) 36 - 40 weeks gestation ("active” hours-blue, type I-“pointed”; bordeaux, type II—“rounded” and green, type IV——mixed”; “quiet” hours—yellow; type III-“flat”).

Table 2. ECG and locomotor activity of the fetus in “quiet” hours.

\begin{tabular}{|c|c|c|c|c|c|c|c|c|c|c|c|c|}
\hline \multirow{3}{*}{ Time (h) } & \multirow{3}{*}{ Type } & \multicolumn{5}{|c|}{ Duration } & \multicolumn{6}{|c|}{ Duration } \\
\hline & & \multicolumn{2}{|c|}{ Oscillation type } & \multicolumn{2}{|c|}{ Fetal movements } & \multirow{2}{*}{$\begin{array}{c}\text { Recording } \\
\text { Min. }\end{array}$} & \multirow{2}{*}{ Time (h) } & \multicolumn{2}{|c|}{ Oscillation type } & \multicolumn{2}{|c|}{ Fetal movements } & \multirow{2}{*}{$\begin{array}{c}\text { Recording } \\
\text { Min. }\end{array}$} \\
\hline & & Min. & Sec. & Min. & Sec. & & & Min. & Sec. & Min. & Sec. & \\
\hline \multirow{4}{*}{$4-5$} & I & - & $20 \pm 0.2$ & - & - & $50 \pm 0.3$ & \multirow{4}{*}{$14-15$} & - & $10 \pm 0.2$ & - & - & $40 \pm 0.4$ \\
\hline & II & $16 \pm 0.3$ & & - & $50 \pm 0$ & & & $3 \pm 0.4$ & & - & $50 \pm 0.1$ & \\
\hline & III & $20 \pm 0.2$ & - & - & - & & & $30 \pm 0.4$ & - & - & - & \\
\hline & IV & $18 \pm 0.2$ & - & - & - & & & $7 \pm 0.1$ & - & & - & \\
\hline \multirow{4}{*}{$5-6$} & I & - & - & $1 \pm 0.1$ & - & $46 \pm 0.2$ & \multirow{4}{*}{$15-16$} & $1 \pm 0.2$ & - & - & - & $54 \pm 0.3$ \\
\hline & II & $7 \pm 0.4$ & - & & - & & & $7 \pm 0.2$ & - & $2 \pm 0.1$ & - & \\
\hline & III & $30 \pm 0.3$ & - & - & - & & & $24 \pm 0.1$ & - & - & - & \\
\hline & IV & $9 \pm 0.3$ & & - & & & & $21 \pm 0.2$ & & & & \\
\hline \multirow{4}{*}{$6-7$} & I & - & $30 \pm 0.2$ & - & - & $59 \pm 0.2$ & \multirow{4}{*}{$16-17$} & - & $20 \pm 0.3$ & - & - & $45 \pm 0.1$ \\
\hline & II & $18 \pm 0.1$ & & 1 & $30 \pm 0.3$ & & & $12 \pm 0.1$ & & $1 \pm 0.3$ & $30 \pm 0.3$ & \\
\hline & III & $26 \pm 0.1$ & - & - & - & & & $21 \pm 0.1$ & - & - & - & \\
\hline & IV & $15 \pm 0.2$ & - & & - & & & $11 \pm 0.1$ & - & - & - & \\
\hline \multirow{4}{*}{$7-8$} & I & - & $50 \pm 0.1$ & - & - & $50 \pm 0.5$ & \multirow{4}{*}{17 - 18} & - & $20 \pm 0.1$ & - & - & $45 \pm 0.3$ \\
\hline & II & $12 \pm 0.4$ & & - & - & & & $8 \pm 0.2$ & & 1 & $50 \pm 0.1$ & \\
\hline & III & $22 \pm 0.4$ & - & 1 & $5 \pm 0.1$ & & & $20 \pm 0.1$ & - & - & - & \\
\hline & IV & $15 \pm 0.3$ & - & - & - & & & $16 \pm 0.1$ & - & & - & \\
\hline \multirow{4}{*}{$8-9$} & I & - & $20 \pm 0.1$ & - & - & $40 \pm 0.2$ & \multirow{4}{*}{$18-19$} & - & $10 \pm 0.1$ & - & - & $42 \pm 0.1$ \\
\hline & II & - & & - & $50 \pm 0.1$ & & & $4 \pm 0.3$ & & - & $40 \pm 0.2$ & \\
\hline & III & $28 \pm 0.3$ & - & - & - & & & $25 \pm 0.2$ & - & - & - & \\
\hline & IV & $12 \pm 0.3$ & - & - & - & & & $12 \pm 0.3$ & - & - & - & \\
\hline
\end{tabular}


Table 3. ECG and locomotor activity of the fetus in "active" hours.

\begin{tabular}{|c|c|c|c|c|c|c|c|c|c|c|c|c|}
\hline \multirow{3}{*}{ Time (h) } & \multirow{3}{*}{ Type } & \multicolumn{5}{|c|}{ Duration } & \multirow{3}{*}{ Time (h) } & \multicolumn{5}{|c|}{ Duration } \\
\hline & & \multicolumn{2}{|c|}{ Oscillation type } & \multicolumn{2}{|c|}{ Fetal movements } & \multirow{2}{*}{$\begin{array}{c}\text { Recording } \\
\text { Min. }\end{array}$} & & \multicolumn{2}{|c|}{ Oscillation type } & \multicolumn{2}{|c|}{ Fetal movements } & \multirow{2}{*}{$\begin{array}{c}\text { Recording } \\
\text { Min. }\end{array}$} \\
\hline & & Min. & Sec. & Min. & Sec. & & & Min. & Sec. & Min. & Sec. & \\
\hline \multirow{4}{*}{$9-10$} & I & - & $45 \pm 0.2$ & $7 \pm 0.2$ & - & $48 \pm 0.2$ & \multirow{4}{*}{$12-13$} & - & $10 \pm 0.2$ & $8 \pm 0.1$ & - & $30 \pm 0.3$ \\
\hline & II & $6 \pm 0.2$ & & & $50 \pm 0$ & & & $10 \pm 0.1$ & & & $50 \pm 0.1$ & \\
\hline & III & $7 \pm 0.3$ & - & - & - & & & $2 \pm 0.1$ & - & - & - & \\
\hline & IV & $35 \pm 0.1$ & - & - & - & & & $17 \pm 0$ & - & & & \\
\hline \multirow{4}{*}{$10-11$} & I & - & $50 \pm 0.4$ & $3 \pm 0.1$ & - & $24 \pm 0.3$ & \multirow{4}{*}{$13-14$} & $2 \pm 0.3$ & - & $4 \pm 0.3$ & - & $48 \pm 0.1$ \\
\hline & II & $8 \pm 0.1$ & & & - & & & $8 \pm 0.1$ & - & & - & \\
\hline & III & $3 \pm 0.1$ & - & - & - & & & $8 \pm 0.2$ & - & - & - & \\
\hline & IV & $12 \pm 0.01$ & $50 \pm 0.1$ & - & - & & & $30 \pm 0.1$ & - & - & - & \\
\hline \multirow{4}{*}{$11-12$} & I & - & $30 \pm 0.2$ & 4 & $50 \pm 0.1$ & $38 \pm 0.2$ & \multirow{4}{*}{$\begin{array}{c}\text { Total from } \\
9-14\end{array}$} & 6 & $45 \pm 0.26$ & 26 & $50 \pm 0.16$ & $188 \pm 0.22$ \\
\hline & II & $10 \pm 0.3$ & & - & & & & $42 \pm 0.16$ & & & & \\
\hline & III & $5 \pm 0.3$ & - & - & - & & & $25 \pm 0.24$ & & & & \\
\hline & IV & $22 \pm 0.2$ & - & - & - & & & 116 & $50 \pm 0.12$ & & & \\
\hline
\end{tabular}

Table 4. ECG and locomotor activity of the fetus in "active” hours.

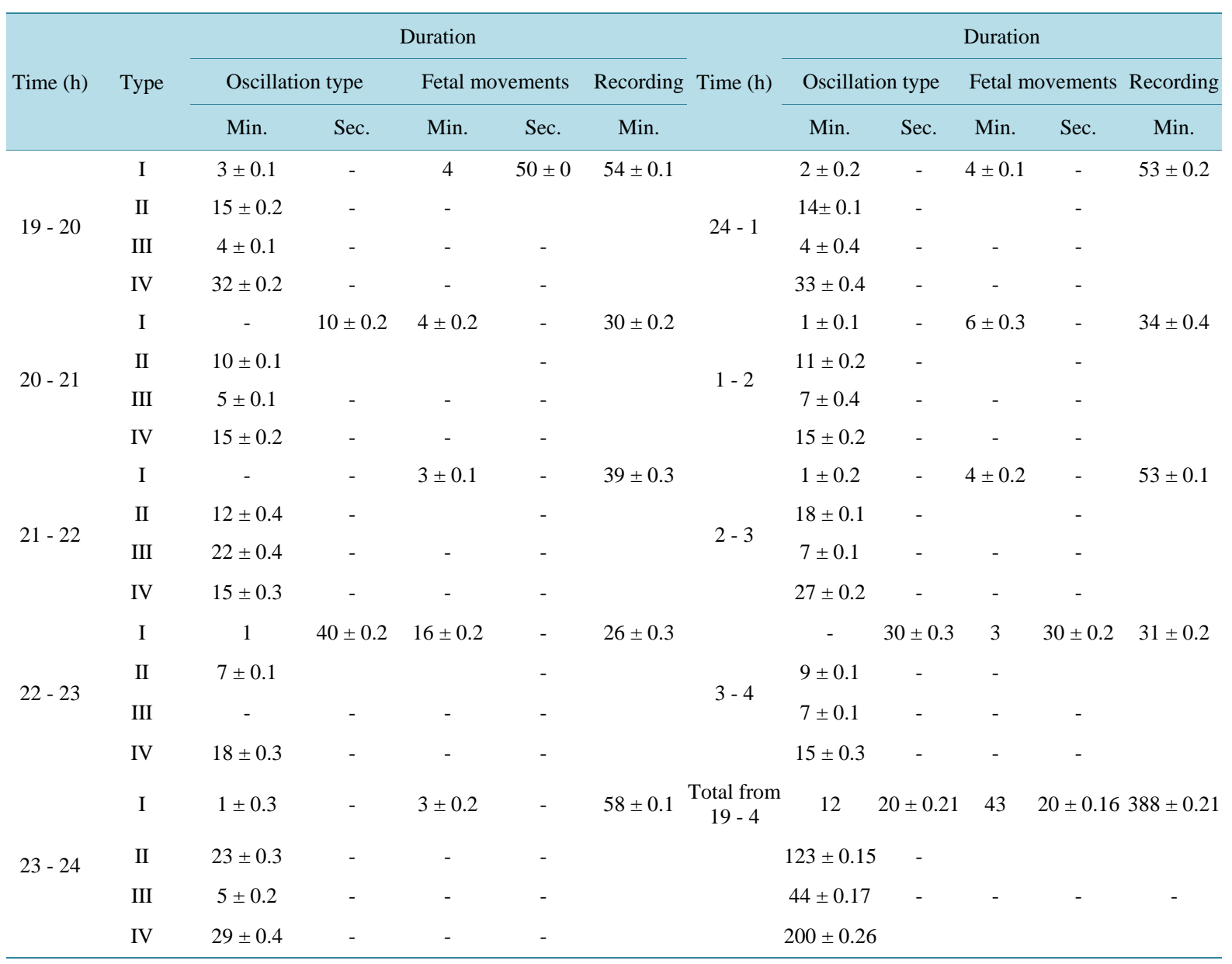




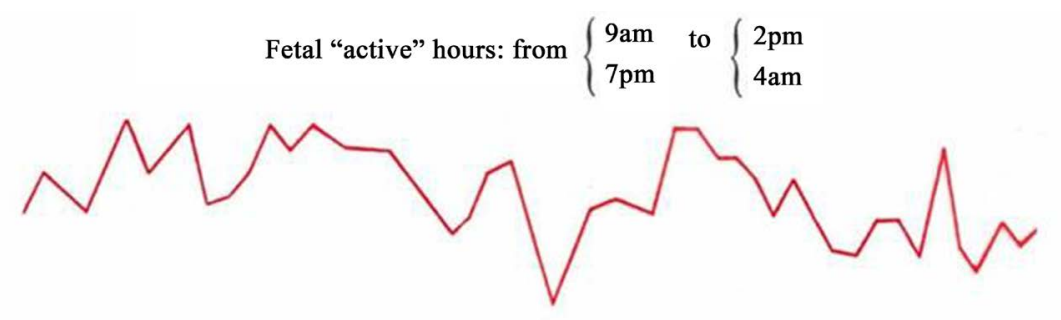

Fetal "quiet" hours: from $\left\{\begin{array}{l}4 \mathrm{am} \\ 2 \mathrm{pm}\end{array}\right.$ to $\left\{\begin{array}{l}9 \mathrm{am} \\ 7 \mathrm{pm}\end{array}\right.$

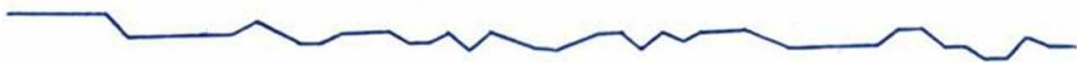

Figure 8. Fetal cardiotachogram “active” and “quiet” hours.

Table 5. Data of concentration “flat” (III) type oscillations in the daily CTG pregnant women.

\begin{tabular}{|c|c|c|}
\hline Time (h) & Duration (min.) & Duration (min. sec.) "flat” type in the CTG \\
\hline $23-24$ & $58^{\prime} \pm 0.1$ & $10^{\prime} 15 " \pm 0.3$ \\
\hline $24-1$ & $53^{\prime} \pm 0.2$ & $16^{\prime} \pm 0.2$ \\
\hline $1-2$ & $34^{\prime} \pm 0.4$ & $19^{\prime} \pm 0.3$ \\
\hline $2-3$ & $53^{\prime} \pm 0.1$ & $19 ' 15 " \pm 0.3$ \\
\hline $3-4$ & $31^{\prime} \pm 0.2$ & $26^{\prime} \pm 0.2$ \\
\hline $4-5$ & $54^{\prime} \pm 0.3$ & $33^{\prime} \pm 0.2$ \\
\hline $5-6$ & $46^{\prime} \pm 0.2$ & $25^{\prime} 5^{\prime \prime} \pm 0.2$ \\
\hline $6-7$ & $59^{\prime} \pm 0.2$ & $25^{\prime} \pm 0.3$ \\
\hline $7-8$ & $50^{\prime} \pm 0.5$ & $18 ' 20 " \pm 0.1$ \\
\hline $8-9$ & $46^{\prime} \pm 0.2$ & $2^{\prime} \pm 0.2$ \\
\hline $9-10$ & $48^{\prime} \pm 0.2$ & $1^{\prime} 10^{\prime \prime} \pm 0.2$ \\
\hline $10-11$ & $24 \pm 0.3$ & $0^{\prime} 50^{\prime \prime} \pm 0.3$ \\
\hline $11-12$ & $38^{\prime} \pm 0.2$ & $0^{\prime}$ \\
\hline $12-13$ & $30^{\prime} \pm 0.3$ & $0^{\prime}$ \\
\hline $13-14$ & $48^{\prime} \pm 0.1$ & $1^{\prime} \pm 0.3$ \\
\hline $14-15$ & $40^{\prime} \pm 0.4$ & $3^{\prime} \pm 0.2$ \\
\hline $15-16$ & $54^{\prime} \pm 0.3$ & $2^{\prime} \pm 0.3$ \\
\hline $16-17$ & $45^{\prime} \pm 0.1$ & $3^{\prime} \pm 0.2$ \\
\hline 17 - 18 & $45^{\prime} \pm 0.3$ & $4^{\prime} \pm 0.2$ \\
\hline $18-19$ & $42^{\prime} \pm 0.1$ & $0^{\prime} 30^{\prime \prime} \pm 0.1$ \\
\hline 19 - 20 & $54^{\prime} \pm 0.1$ & $3^{\prime} \pm 0.2$ \\
\hline $20-21$ & $30^{\prime} \pm 0.2$ & $2 ' 30 " \pm 0.2$ \\
\hline $21-22$ & $39^{\prime} \pm 0.3$ & $2^{\prime} \pm 0.2$ \\
\hline $22-23$ & $26^{\prime} \pm 0.3$ & $2^{\prime} \pm 0.2$ \\
\hline
\end{tabular}




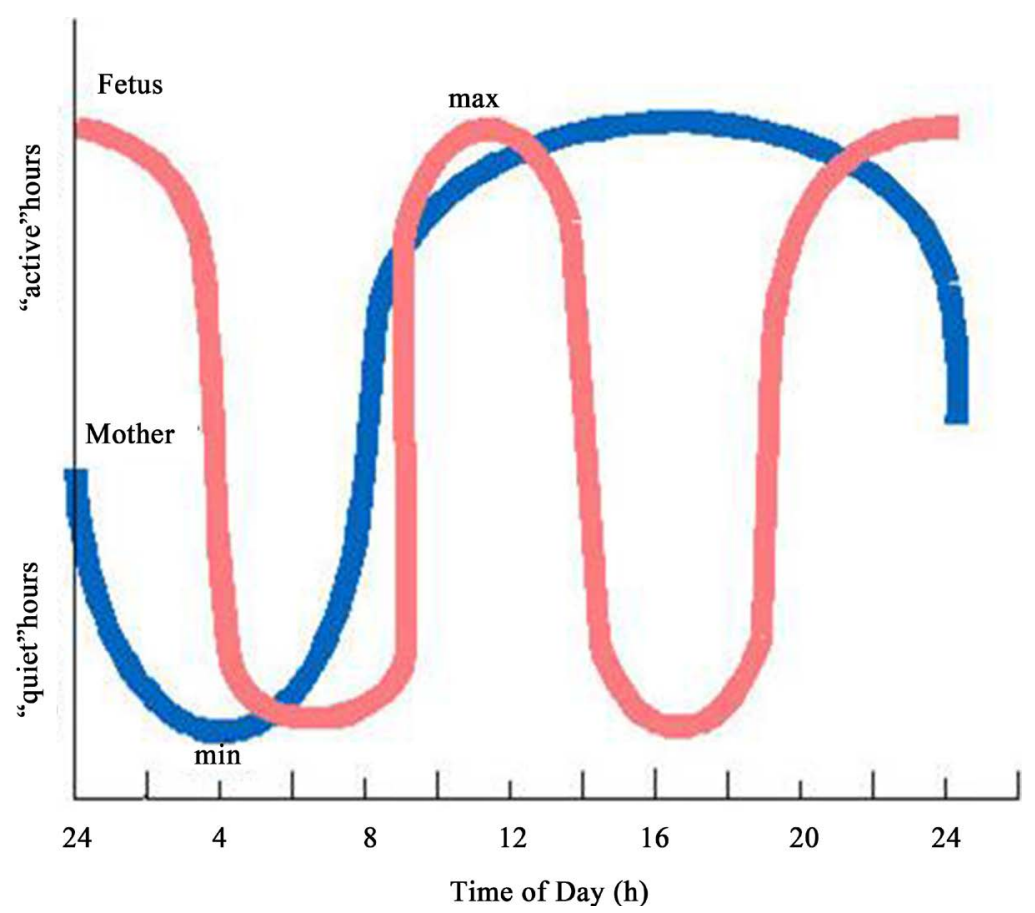

Figure 9. The diagram shows the biorhythms of coordinated maternal (blue line) and fetal (red line) cardiac activity revealed in our study. On the abscissa-time (hours of the day), on the coordinate-periods of the day ("active" and "quiet" hours).

of the types of oscillation in "active" and "passive" hours of the fetus revealed the reliable result $(\mathrm{P}<0.001)$.

Analysis of the motion activity of the fetus during 24 hours revealed that duration of motions during "passive" hours for one hour of registration did not exceed $1 \mathrm{~min} .50 \mathrm{sec}$. and for the whole period of "passive" hours constituted $11 \mathrm{~min}$. $5 \mathrm{sec}$. which corresponds to $2.3 \%$. Motions of the fetus during "active" hours for one hour of registration constituted more than three minutes, achieving the maximal point of $32 \mathrm{~min} . \pm 0.2$ during the period of time from 2200h to 2300h. Motions of the fetus during the whole period of "active" hours were registered during 91 min. 10 sec., which constituted $16 \%$ of total time.

Apart from the above analysis, we decided to calculate the number of heartbeats of the fetus lower than 120 beats/min. and exceeding 160 beats/min., expressed on the daily hourly CTG. See the obtained data in the Table 6.

The data obtained indicate a significant prevalence of periods of frequencies lower than 120 beats/min in "passive" hours of the fetus, while frequencies exceeding 160 beats/min either were not registered or were solitary.

Thus, our studies reveal that "active" periods of the fetus are characterized by the increased level of cardiovascular system functioning, which is expressed by the prevalence of "pointed", "round" and "mixed" types of oscillation with high level of intra-minute fluctuations and variability of FHR; prevalence of frequencies over 160 beats/min and increased motion activity of the fetus (Figure 10).

"Passive" periods are characterized by decreased variability of FHR, prevalence of "flat" (III) type of oscillation, significant prevalence of frequencies lower than 120 beats/min and drastic deceleration of motion activity. We can suggest that within the period of 4 a.m. - 9 a.m. and 2 p.m. - 7 a.m., the fetus expresses "hypnoid condition".

Correspondingly, similarly to an adult individual, the fetus suffers interchange of periods of increase and decrease of physiological functions. The curve of the dynamics of the alteration of the level of functioning of the physiological systems has a biphasic character with a decrease during the morning hours (from 4 a.m. to 9 a.m.) and during the midday (from $14 \mathrm{~h} 00$ to 19h00) and with increase during the daytime (from 9 a.m. to 2 p.m.) and during the evening and night (from 7 p.m. to 4 a.m.) [20]-[36].

According to many of the authors, alterations of most of the human physiological processes (temperature of 
Table 6. The number of FHR of $120 \mathrm{bpm}$ and $150 \mathrm{bpm}$ during the day.

\begin{tabular}{|c|c|c|c|}
\hline \multirow{2}{*}{ Time (h) } & \multirow{2}{*}{ Duration (min.) } & \multicolumn{2}{|c|}{ The number of FHR } \\
\hline & & Below 120 bpm & Above $150 \mathrm{bpm}$ \\
\hline $23-24$ & $58^{\prime} \pm 0.1$ & 6 time \pm 2.4 & 57 time \pm 2.1 \\
\hline $24-1$ & $53^{\prime} \pm 0.2$ & 8 time \pm 1.8 & 41 time \pm 2.0 \\
\hline $1-2$ & $34^{\prime} \pm 0.4$ & 28 time \pm 2.2 & 0 time \\
\hline $2-3$ & $53^{\prime} \pm 0.1$ & 25 time \pm 2.1 & 0 time \\
\hline $3-4$ & $31^{\prime} \pm 0.2$ & 14 time \pm 2.4 & 1 time \pm 0.8 \\
\hline $4-5$ & $54^{\prime} \pm 0.3$ & 52 time \pm 2.0 & 2 time \pm 1.2 \\
\hline $5-6$ & $46^{\prime} \pm 0.2$ & 75 time \pm 1.8 & 0 time \\
\hline $6-7$ & $59^{\prime} \pm 0.2$ & 80 time \pm 1.9 & 0 time \\
\hline $7-8$ & $50^{\prime} \pm 0.5$ & 10 time \pm 2.4 & 0 time \\
\hline $8-9$ & $46^{\prime} \pm 0.2$ & 2 time \pm 1.6 & 0 time \\
\hline $9-10$ & $48^{\prime} \pm 0.2$ & 3 time \pm 1.8 & 5 time \pm 1.7 \\
\hline $10-11$ & $24^{\prime} \pm 0.3$ & 3 time \pm 2.3 & 4 time \pm 1.6 \\
\hline $11-12$ & $38^{\prime} \pm 0.2$ & 2 time \pm 1.9 & 29 time \pm 1.7 \\
\hline $12-13$ & $30^{\prime} \pm 0.3$ & 1 time \pm 0.9 & 40 time \pm 2.4 \\
\hline $13-14$ & $48^{\prime} \pm 0.1$ & 13 time \pm 1.7 & 4 time \pm 2.1 \\
\hline $14-15$ & $40^{\prime} \pm 0.4$ & 22 time \pm 2.3 & 0 time \\
\hline $15-16$ & $54^{\prime} \pm 0.3$ & 26 time \pm 1.9 & 0 time \\
\hline $16-17$ & $45^{\prime} \pm 0.1$ & 33 time \pm 1.7 & 9 time \pm 0.9 \\
\hline $17-18$ & $45^{\prime} \pm 0.3$ & 37 time \pm 1.9 & 5 time \pm 1.2 \\
\hline $18-19$ & $42^{\prime} \pm 0.1$ & 25 time \pm 2.3 & 5 time \pm 1.7 \\
\hline $19-20$ & $54^{\prime} \pm 0.1$ & 13 time \pm 2.1 & 36 time \pm 2.2 \\
\hline $20-21$ & $30^{\prime} \pm 0.2$ & 31 time \pm 1.9 & 22 time \pm 1.5 \\
\hline $21-22$ & $39^{\prime} \pm 0.3$ & 14 time \pm 1.6 & 38 time \pm 2.8 \\
\hline $22-23$ & $26^{\prime} \pm 0.3$ & 0 time & 23 time \pm 2.0 \\
\hline
\end{tabular}

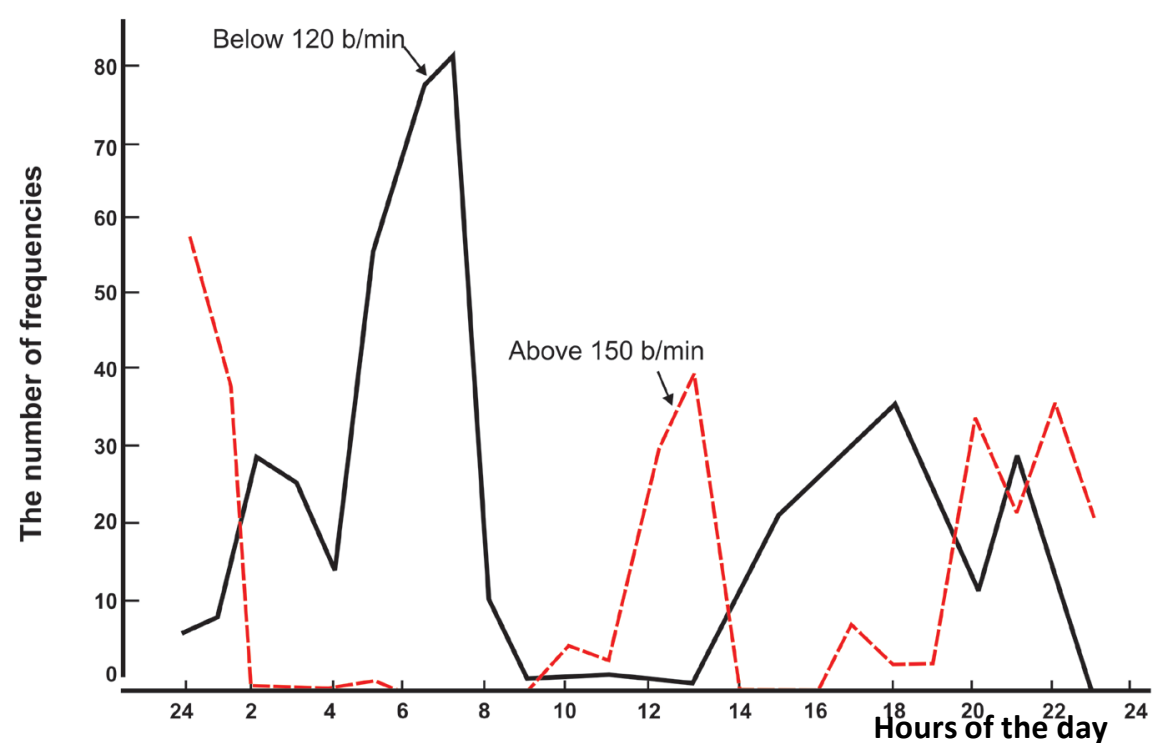

Figure 10. The concentration of frequencies below 120 beats per minute (solid line) and above 150 beats per minute (interrupted) during the day in the fetus. On the horizontal axis-time (day time), the vertical axis-the number of frequencies (on the tens and units). 
the body, activity of cardiovascular system, respiration etc.) are expressed in constant increase of the level thereof from $08 \mathrm{~h} 00$ to $13 \mathrm{~h} 00$ and then from $13 \mathrm{~h} 00$ to $14 \mathrm{~h} 00$ they slightly decreased to re-increase achieving the maximal point at $16 \mathrm{~h} 00-18 \mathrm{~h} 00$. The second minimum of these parameters can be detected within the period of 02h00 - $03 \mathrm{~h} 00 \mathrm{a}$ am. In the adverse phase, the daily rhythm of the amount of adrenaline in the blood and activity of hypophyseal-adrenal system vary with peaks at 06h00 - 09h00.

Melatonin, as a rule, is high during the nighttime and low during the day. Normal standard of melatonin in hormonal studies on blood constitutes $10-20 \mathrm{ng} / \mathrm{ml}$ during the daylight and $80-100 \mathrm{ng} / \mathrm{ml}$ during the night. Half-disintegration period of melatonin constitutes 45 minutes, which means that tests on the hereof hormone shall be held immediately after blood sampling, at that it is necessary to indicate the exact time of sampling of bio-material. Endogenous secretion of growth hormone (GH) is of undulatory character. Maximal point of the upcoming peak of GH is registered every three-five hours. As a result, we can averagely witness 6 - 10 increases and decreases of concentration of GH during 24 hours. The peak of highest amplitude of secretion of GH is registered during night in one or two hours upon falling asleep and lasts approximately two hours successively. Maximal point of thyroxin [T4] can be detected during the period of $08 \mathrm{~h} 00$ - 12h00, while the minimal pointduring the night time, within 23h00 - 03h00. Besides, maximal amount of T4 can be registered during autumnwinter time, while the minimal amount = during spring-summer period. Exception constitutes pregnancy till the III trimester, when the indices of T4 (general) and T3 (general) can be high regardless of the period of time or weather conditions. T3 (free), on the contrary, is decreased to the III trimester.

Prolactin has a clearly expressed circadian rhythm of section with maximal emission of hormone during the night sleep. During the lutein phase of the menstrual cycle, the level of prolactin is higher than during the follicular phase etc.

Secretion of Lutein hormone of women in reproductive period, is directly linked with the phase of menstrual cycles. The highest level of LH is registered in the middle of the cycle (ovulation period) and the lowest level in the end of the follicular phase.

Progestaron (HR) is the intermediate member in synthesis of androgens. The maximal level of secretion constitutes 5 - 7 days after ovulation. In the event of non-fertilization, the level of HR is decreased and on the contrary, in the event if the fertilized ovum is being implanted, then the corpus luteum continues secretion of the highest amount of HR till the $12^{\text {th }}$ week of pregnancy.

The highest secretion of testosterones of men is registered during the early morning hours after awakening within the period of $06 \mathrm{~h} 00$ - 07h00. At $09 \mathrm{~h} 00$ - 11h00, the basal level of testosterone is stabilized, continuing light secondary fluctuations (with frequency of 5 - 9 times per one hour). At 18h00, another peak of production of testosterone is registered at $09 \mathrm{~h} 00-10 \mathrm{~h} 00 \mathrm{pm}$, substituted with cascade decrease [7] [12] [25] [37]-[42].

The researchers lead us further to particular problems and issues, attracting our attention to the molecular organization of living matter. The hereof studies are very valuable and necessary. However, organism, as a whole, disappears from our field of vision to an increasing extent.

Our surveys have been dedicated to study of bio-rhythms of heart activity and motion activity of the fetus, to detect inter-dependency of bio-rhythms of a pregnant woman and a fetus.

Our studies have resulted to detection of bio-rhythms of sleep and vigilance of intrauterine fetus. We have revealed mechanisms of inter-dependency of two organisms: mother and fetus. The studies we've held revealed importance of the leading, fundamental, dominating bio-rhythm of sleep and vigilance, the bio-rhythm, which is generated and coordinated by the UNIVERSE.

All the systems and functions of a healthy organism are in concord with the bio-rhythms of sleep and vigilance. Disturbance entails not only disorder of rhythms and functionality of some separate organs and systems but it leads to violation of rhythms of fast wave and slow wave sleep.

Hence, we can hereby conclude that the periods of time from $04 \mathrm{~h} 00$ to $09 \mathrm{~h} 00$ and from $14 \mathrm{~h} 00$ to $20 \mathrm{~h} 00$ are the periods of transition of organism of the pregnant woman from one level of functionality to another, which is a natural functional load not only for organism of the mother but for the fetus as well.

We can presume that the fetus, in the curse of intrauterine development, needs relatively constant homeostasis and expressing own adaptive reactions to rhythmically altering level of functioning of organism of the pregnant woman, the fetus is activated in the period of decrease of physiological functions of the mother and is exposed to "hypnoid" condition upon increase of these functions of the pregnant woman.

Experimental studies of Arkhangelskaya N.A. [18] intercross with our data. The author has defined feebly marked daily rhythm of temperature of the body of the fetus according to its configuration, adverse to the 
rhythm of the mother and explained formation of thermoregulatory conditional reflex, related to decrease of temperature of the body of the mother during the nighttime.

According to various researches, alterations of most of the physiological processes in human organism (temperature of the body, activity of cardio-vascular system, breath etc.) are expressed in constant increase of their level from $08 \mathrm{~h} 00$ to $13 \mathrm{~h} 00$ and then from $13 \mathrm{~h} 00$ to $14 \mathrm{~h} 00$ - are slightly reduced to be increased again achieving maximal point to $16 \mathrm{~h} 00$ - 18h00. The second minimum of these temperatures are registered within $02 \mathrm{~h} 00$ $03 \mathrm{~h} 00 \mathrm{am}$. On anti-phase, the daily rhythm of concentration of adrenalin in the blood, activity of hypophysial-adrenaline systems with peaks at 06h00 - 09h00 fluctuate.

Studying the characteristics of reaction of the fetus to the functional probes, taking the defined biological rhythms of the fetus into account, we held studies in "active" and "passive" (or hypnoid) periods.

\subsection{Definition of the Functional Condition of the Fetus by Means of Control Tests in "Active" Hours}

The background CTG of the fetus in "active" hours was expressed with "mixed" (IV) type of oscillation. Analysis revealed that the data of the background CTG fail to give comprehensive information to allow formation of the maximal picture of the intrauterine condition of the fetus. Many of the authors agree on lack of comprehensive information by the background CTG insufficient to characterize the condition of the fetus. As to the intra-minute fluctuations, then most of the researches attach utmost importance thereto and classify the types of CTG according to the degree of deviation of FHR of the fetus from the basic (background) rhythm, allowing define the pathologic condition thereof. Results of our studies contradict with these data. Intra-minute fluctuations, similarly to other indices of the background CTG of the fetus, fail to satisfy as the medical personnel so the pregnant woman.

We, upon application of the functional loads for definition of the condition of the fetus in "active" hours, have classified three types of reactions as: "acceleration", "deceleration" and "zero". In 94\% of cases, in the group of the fetuses with the reaction type "acceleration", we registered high estimation according to Apgar scale (8 - 10 points). The latent period of reaction of the fetus to the probe constituted $1.2 \pm 02 \mathrm{sec}$. (sub-group A) and $1.9 \pm$ 0.7 sec. (sub-group B). Recovery time of FHR to the initial point in the sub-group A constituted-35, $0 \pm 2.6$ sec. and-39, $0 \pm 3.1$ sec. in the sub-group $B$.

In 76.2\% of cases upon reaction type "deceleration" of the fetus, estimation according to Apgar scale constituted 8 - 10 points. And in $23.8 \%$ of cases -7 - 8 points. Latent period constituted: in the sub-group A-1.3 \pm $0.4 \mathrm{sec}$. and in the sub-group B-1.9 $\pm 0.9 \mathrm{sec}$. Recovery time of FHR to the initial point constituted-37.0 \pm 3.4 sec. (sub-group A) and $39.0 \pm 3.6$ sec. (sub-group B).

$48.2 \%$ of newborns were born in the group of the fetuses with the reaction type "zero" with high estimation on Apgar scale. In 51.8\% of cases, estimation on Apgar scale constituted 5 - 8 points. Pre-natal fetal death was detected in seven cases (in two cases-accident) and in five cases-death of newborns. In $32.6 \%$ of cases, various disorders of early neonatal period were detected.

The basal rhythm of the functional load of the fetus, born with high estimation according to Apgar scale, constituted $136.8 \pm 2.1$ beats/sec. and the basal rhythm of the functional load of the fetus, born with estimation lower than 8 points according to Apgar scale-157.4 \pm 3.2 beats/sec.

According to many authors, acceleration of FHR in response to the load is the index of the satisfactory condition of the fetus et al. declare that deceleration of FHR upon the tests indicates to disturbance of intrauterine condition of the fetus and decrease of adaptive reactions thereof. Stimulus of various objects of study entails various reactions in dependence on the characteristics of the nervous system, as well as in dependence on functional condition of organism upon studies. Correspondingly, various types of reactions of the fetuses to the test can be demonstration of various levels of maturity of sympathetic and parasympathetic nervous systems, as well as they can depend on the condition of the functional systems of the mother and the fetus upon studies.

On the basis of our data, we can conclude that the healthy fetus, in "active" hours, reacts on functional load with acceleration or deceleration of FHR with short latent periods, swift recovery to the initial point and maintenance of the basal rhythm after tests. The best reaction of the fetus is of the type "acceleration". Slight extension of latent period for the fetuses, composing the sub-group B is to be paid attention in comparison with the fetuses of the sub-group A, which indicates to the negative impact of the pathologic pregnancy to the fetus and can be used as the earliest sign of deviation of the fetal condition. 
The fetuses attract the highest attention with stabile rhythm-that is, "zero" type of reaction to the test. This type of reaction, registered in "active" hours, indicates to decrease of compensation abilities of the fetus and is, as we consider, the index of the transitional condition from healthy to pre-pathologic period.

According to D. Navot [41] [43], N. Pereira-Lur et al. [42] absence of reaction of the fetus upon functional probes is estimated as pathologic phenomenon, certifying intrauterine hypoxia of the fetus [5] [44] [45].

In view to define the diagnostic value of the functional loads upon the combined application thereof, we have analyzed CTG of 800 fetuses. Studies were held in "active" hours of the fetus. We were to define the diagnostic value of six control tests, each of which implied the complex of two or three tests, applied in dynamics with one record of external CTG of the fetus in view of definition of intrauterine condition of the fetus. Number of fetuses that we exposed to examination according to each complex of control tests is as follows:

1) Exercise stress and audio stimulus: 150 pregnant women altogether (sub-group A-75; sub-group B-75);

2) Breath-holding upon exhalation and audio stimulus: 150 pregnant women altogether (sub-group A—75; sub-group B-75);

3) Breath-holding upon exhalation and exercise stress: 100 pregnant women altogether (sub-group A—50; sub-group B-50);

4) Audio stimulus and NST: 100 pregnant women altogether (sub-group A-50; sub-group B-50);

5) Exercise stress and NST: 100 pregnant women altogether (sub-group A-50; sub-group B-50);

6) Audio stimulus, exercise stress and NST: 200 pregnant women altogether (sub-group A-100, sub-group B-100).

a) Exercise stress and audio stimulus-type "acceleration" in response to both loads was registered in 81 (54\%) cases (sub-group A—59 fetuses ,72\%); sub-group B-22 fetuses (27\%). 79 fetuses were born with high estimation according to Apgar scale-8 - 10 points, newborn period was estimated as satisfactory, women were discharged from hospital after 8 - 9 days. In two cases, estimation according to Apgar scale was 7 - 8 points, women were discharged after 11 days. Cases of reaction of the fetus to both loads on the type of "deceleration" were not registered. In 18 cases (12\%), "zero" reaction of the fetus (sub-group A-6 fetuses-33.3\%, sub-group B-12 fetuses-66.7\%) was registered. In one case (5.5\%) we registered high estimation according to Apgar scale-8 - 9 points. Newborn period did not reveal aggravations, women were discharged from hospital after 9 days. In 17 cases (94.5\%), estimation according to the Apgar scale constituted 7 - 8 points: early neonatal period of newborns revealed disturbance of adaptation processes-slow weight gain, pale skin (women were discharged after 12 days), and condition of 5 fetuses was estimated as moderately grave. Women were discharged after 14 15 days. In 51 cases (34\%) out of 150, various types of reactions of one and the same fetus were registered in response to exercise stress and audio stimulus (sub-group A-10, sub-group B-41). In 29 cases (56.8\%), the fetuses expressed reactions of the type "acceleration" to one of the loads while others expressed reaction to the type "deceleration". 19 cases out of them expressed reaction of type "deceleration" to the exercise stress and reaction of the type "acceleration" to the audio stimulus. In 10 cases, reaction of the type "acceleration" was expressed to the exercise stress and the reaction of type "deceleration" was expressed to the audio stimulus. In 28 cases (96.5\%), estimation according to Apgar scale constituted 8 - 10 points, newborn period did not reveal any aggravations. In one case (3.5\%), estimation according to Apgar scale constituted 6 - 7 points ("acceleration” to the exercise stress and "deceleration" to the audio stimulus, neonatal period revealed disturbances of adaptation period. Women were discharged after 12 days). In 22 cases (16\%), "zero" type of reaction was expressed to one of the loads and "acceleration" or "deceleration" to another. In 15 cases out of them, the fetuses expressed reaction "acceleration" (9 cases) or "deceleration" (6 cases) to the audio stimulus and "zero" reaction to the exercise stress. In 14 cases (93.7\%), estimation according to Apgar scale constituted 8 - 10 points, newborn period did not reveal any aggravations. In one case, estimation according to Apgar scale was lower than 8 points and in early neonatal period we detected disturbances of adaptation period. In 7 cases, "zero" reaction was expressed to the audio stimulus and "acceleration" (3 cases" or "deceleration" (4 cases) to the exercise stress. In all 7 cases, estimation according to Apgar scale was lower than 8 points and newborn period of two children revealed various disturbances of adaptation period and the condition of five children was estimated as moderately grave. The index of reactivity of the fetus to combined application of exercise stress and audio stimulus constituted 88\% and diagnostic evaluation-92\%.

b) Breath-holding upon exhalation and audio stimulus. Reaction of the fetus to both loads was "acceleration" in 36 cases-24\% (sub-group A-31 cases, sub-group B-5 cases). Estimation according to Apgar scale constituted 8 - 10 points in 29 cases (80.5\%), newborn period of these children was estimated as physiological. In other 7 cases (19.4\%), estimation according to Apgar scale was lower than 8 points and the condition of the child- 
ren in newborn period was estimated as moderately grave. In 9 cases-6\%-we detected reaction of type "deceleration" to both loads (sub-group A). High estimation according to Apgar scale was registered in all 9 cases, newborn period was physiological. "Zero" reaction of the fetus to these loads was expressed in 23 cases-15.3\% (sub-group A-14 cases; sub-group B-9 cases). High estimation according to Apgar scale was registered in 17 cases (73.9\%), newborn period expressed no aggravations. In 6 cases (26.1\%), estimation according to Apgar scale constituted 6 - 7 points, newborn period of four children was estimated as moderately grave and two children revealed symptoms of disturbance of adaptation. In 16 cases (10.6\%), "acceleration” was registered to breath-holding upon exhalation and "deceleration" to the audio stimulus. Newborn period of 8 - 10 points was registered in 9 cases (56.3\%), newborn period of these fetuses was physiological. In 7 cases (43.7\%), estimation according to Apgar scale was lower than 8 points and early neonatal period revealed symptoms of disturbance of adaptation. 72 fetuses (18\%) expressed reaction "deceleration" to breath-holding upon exhalation and "acceleration" to the audio stimulus. Estimation according to Apgar scale in 23 cases (85\%) constituted 8 - 10 pointsnewborns were in favorable state and discharged. In 4 cases (14.8\%) estimation according to Apgar scale was lower than 8 points; condition of the children in early neonatal period was estimated as moderately grave. In 8 cases, "acceleration" (5 cases) or "deceleration" (3 cases) was expressed to breath-holding upon exhalation and "zero" type reaction was expressed to the audio stimulus. High estimation according to Apgar scale was registered in three cases (37.5\%) - newborns were in favorable state and discharged in 8 - 9 days after birth. In 5 cases (62.5\%), estimation according to Apgar scale was lower than 8 points, and in one case of early neonatal period-physiological—4 cases-disturbance of adaptation processes. In 31 cases (20.7\%), "zero" type of reaction was expressed to breath-holding upon exhalation and "acceleration" (27 cases) or "deceleration" (4 cases) to audio stimulus. High estimation according to Apgar scale was registered in 25 cases (80.6\%), newborn period was physiological and in 6 cases (19.4\%), estimation according to Apgar scale constituted 7 - 8 points. Only two children revealed symptoms of disturbance of adaptation. The index of reactivity of the fetus to control tests constituted $84.6 \%$ and diagnostic value- $69.3 \%$.

c) Breath-holding upon exhalation and exercise stress: in 100 cases (sub-group A-50, sub-group B-50), breath-taking upon exhalation and exercise stress were applied in capacity of functional loads. The type "acceleration", in response to both stresses, was registered in 19 cases-19\% (sub-group A-12, sub-group B-7). Estimation according to Apgar scale of 8 - 10 points was expressed in 15 newborns (79.0), newborn period of the hereof children was physiological and the mothers were discharged after 8 - 9 days in satisfactory condition. In other four cases (21\%), estimation according to Apgar scale was lower-8 points, newborn period revealed the symptoms of disorder of adaptation, and the health state of two children were of average gravity (estimation according to Apgar scale was 6 - 8 points). The mothers were discharged after 16 days.

The type "deceleration", in response to both stresses, was registered in 13 cases-13\% (sub-group A-10, sub-group B-3). Estimation according to Apgar scale was 8 - 10 points, newborn period was physiological. In other five cases (38.4\%), estimation according to Apgar scale was 6 - 7 points and the health state of the newborn children was evaluated as of average gravity. The type "zero" reaction of the fetus in response to both stresses was registered in 28 cases-28\% (sub-group A-19, sub-group B-9). In 21 cases out of them (75\%), estimation according to Apgar scale was 8 - 10 points and in 7 cases (25\%) - 5 - 7 points. The state of six children out of 7 born with low estimation according to Apgar scale, was evaluated as of average gravity, and the health state of one child was evaluated as grave (the mother was discharged after 21 days). In 40 cases, upon two stresses held in dynamics with one and the same record and one and the same fetus, various types of reactions have been registered. In 9 cases (22.5\%), in response to breath-holding upon exhalation, the reaction of the fetus was "acceleration" and in response to the exercise stress, the reaction was of the type "deceleration". Estimation according to Apgar scale of 8 fetuses amongst them (88.8\%) was 8 - 9 points and in one case (11.2\%) 7 points. Newborn period of all the children was physiological. Reaction of 6 fetuses (15\%) in response to breath-holding upon exhalation was of the type "deceleration" and in response to the exercise stress- "acceleration". Escalation according to Apgar scale of all six cases was 8 - 9 points, newborns were in satisfactory state and discharged after 9 days. In 25 cases (62.5\%), reaction of the fetuses in response to one of the tests was of "zero" type. In 17 cases (68\%), "zero" type reaction was registered upon the test of breath-holding at exhalation and reaction in response to the exercise stress was of "acceleration" type (11 cases) or "deceleration" type (6 cases). In 17 cases out of 16 (94.1\%), estimation according to Apgar scale was 8 - 10 points, and the mothers were discharged after 8 - 9 days in satisfactory condition. In one case (5.9\%), estimation according to Apgar scale was 7 points, the newborn, in an early neonatal period, expressed signs of disturbance of adaptation process. In 8 cases (32\%), in response to breath-holding upon exhalation, reaction of "acceleration" type was registered (5 
cases) or was registered "deceleration" type (3 cases), and in response to the exercise stress, type "zero" was registered. Estimation according to Apgar scale was 8 - 10 points in 4 cases (50\%) and during the newborn period, it was physiological. In 4 cases, estimation according to Apgar scale was 6 - 7 points; there newborn expressed various symptoms of disturbance of adaptation. Index of reactivity of the fetus to the control tests constituted $72 \%$, and diagnostic value of the test constituted $64 \%$.

d) Audio stimulus and NST: in 100 cases, the audio stimulus and NST were applied in capacity of tests (sub-group A-50, sub-group B-50). The "acceleration" type, in response to both stresses, was registered in 27 cases (27\%) (sub-group A-18, 36\%; sub-group B-9, 18\%). Estimation according to Apgar scale was 8 - 10 points. 26 fetuses (96.3\%) were born. Newborn period was physiological without aggravations. In one case, estimation according to Apgar scale was 7 points, signs of disturbance of adaptation period were registered during the newborn period. "Deceleration" type was registered in 14 cases-14\% (sub-group A-11, 22\%; sub-group B-3, 6\%). High estimation according to Apgar scale was 8 - 20 points, registered in 9 cases (64.3\%); newborn period was physiological. In 5 cases (35.7\%), estimation according to Apgar scale was 7 points and in early neonatal period were expressed symptoms of disturbance of adaptation period. in 8 cases (8\%), "zero" type of reaction of the fetus was registered in response to the audio stimulus and NST (sub-group A-2, 36\%; sub-group B-6, 6\%). Estimation according to Apgar scale was 8 - 10 points in 3 cases (37.5\%). Newborn period was physiological. Mothers were discharged after 9 days in satisfactory state. In 5 cases (62.5\%), estimation according to Apgar scale was 6 - 7 points. Health state of the newborns was estimated as averagely grave (4 cases) and as grave in one case. In 51 cases (51\%) out of 100, various types of reaction of one and the same fetus were expressed in response to audio stimulus and NST. In 14 cases (27.5\%), “deceleration” type was registered in response to NST and "acceleration" type was registered in response to audio stimulus. High estimation according to Apgar scale was 8 - 10 points in 14 cases; newborn period was physiological. In 11 cases (21.6\%), reaction of the fetus in response to NST was "acceleration" and in response to audio stimulus- "deceleration". Estimation according to Apgar scale was 8 - 9 points in 7 cases (63.6\%), newborns, in satisfactory health state, were discharged after 8-9 days. In 4 cases (36.3\%), estimation according to Apgar scale was 6 - 7 points; health state of newborns was estimated as of average gravity (3 cases) as grave in one case. In 9 cases (17.6\%), reaction of the fetus in response to the audio stimulus was of "zero" type, and reaction in response to NST was "acceleration type in 4 cases and "deceleration" type in 5 cases. Estimation according to Apgar scale was 8 - 9 points in 4 cases (44.5\%); newborns were in satisfactory health state and discharged. In 5 cases (55.5\%), estimation according to Apgar scale was 6 - 7 points, the health state of newborns in early neonatal period was estimated as averagely grave. 17 fetuses (33.3\%) expressed reaction of type "zero" in response to NST and type "acceleration in response to audio stimulus (12 cases), and "deceleration" (5 cases). Estimation according to Apgar scale was 8 - 9 points in 10 cases (58.8\%), newborns were in satisfactory health state and discharged after 8 - 9 days. Estimation according to Apgar scale of 6 newborns (35.3\%) was 7 - 8 points, newborn period was physiological. In one case (5.9\%), intranatal spontaneous fetal death was registered ( $4^{\text {th }}$ delivery) entailed with short umbilical cord $(37 \mathrm{~cm})$, true knot. Index of reactivity of the fetus in response to control tests constituted $92 \%$, and diagnostic value constituted $75 \%$.

e) Exercise stress and NST: in 100 cases, we have applied exercise stress and NST in capacity of the tests in view of identification of intrauterine state of the fetus (sub-group A-50, sub-group B-50). The "acceleration" type in response to both stresses was registered in 32 cases-32\% (sub-group A-23, 46\%; sub-group B-9, 18\%). High estimation according to Apgar scale-8 - 9 points-was registered in 27 cases (84.4\%), newborns, in satisfactory health state, were discharged after 8 - 9 days. 5 newborns (15.6\%) were born with low estimation according to Apgar scale- 8 points; disturbance of adaptation processes was expressed by 3 newborns; the health state of two children was estimated as averagely grave. "Deceleration" type was registered in 7 cases-7\% (sub-group A-6, 12\%; sub-group B-1, 2\%). 7 newborns were born with estimation of 8 - 10 points according to Apgar scale; newborn period was physiological. "Zero" type of reaction was expressed in 15 cases-15\% (sub-group A-2, 4\%; sub-group B-13, 26\%). High estimation according to Apgar scale-8 - 10 points—was registered in 8 cases (53.3\%), newborn period expressed no aggravations. 7 fetuses (46.7\%) were born with estimation of $6-7$ points according to Apgar scale, symptoms of disturbance of adaptation processes in neonatal period were expressed in 3 newborns, and health state of 4 children was estimated as averagely grave.

In 46 cases (46\%), in response to the exercise stress and NST, one and the same fetus expressed various types of reaction. In 15 cases (32.6\%), in response to NST, reaction of the fetuses was of "deceleration" type and in response to exercise stress- "acceleration" type. Estimation according to Apgar scale-8 - 10 points-was registered in 11 fetuses (73.3\%), newborn period was physiological. In 4 cases (26.7\%), estimation according to 
Apgar scale was 6 - 7 points; in one case, newborn period was expressed with the symptoms of disturbance of adaptation period, in two cases, the health state of the children was estimated as averagely grave and in one case as grave.

In 22 cases (47.8\%), in response to NST, reaction of the fetuses was of “acceleration” type and reaction in response to the exercise stress was of "deceleration" type. Estimation according to Apgar scale— 8 - 10 pointswas registered in 22 fetuses, and newborn period was physiological. In 7 cases (15.2\%), upon application of both stresses, reaction of the fetus in response to NST was "acceleration" (6 cases, 85.7\%) or "deceleration" (1 case, $14.3 \%$ ), and in response to the exercise stress_- “zero” type. High estimation according to Apgar scale-8 9 points-was registered in 5 fetuses (71.4\%), newborn period was physiological. In 2 cases (28.8\%), low estimation according to Apgar scale was expressed in 6 - 7 points; the health state of the children in early neonatal period was estimated as averagely grave. In 2 cases (4.42\%), in response to NST, the reaction of the fetus was of "zero" type and in response to the exercise stress— “acceleration". In both cases, estimation according to Apgar scale was 8 - 9 points; newborns were discharged in satisfactory state.

Index of reactivity of the fetus in response to the control tests constituted $85 \%$, and diagnostic value constituted $81 \%$.

f) Audio stimulus, exercise stress and NST. In 200 cases (sub-group A-100, sub-group B-100), we have applied audio stimulus, exercise stress and NST in capacity of the functional stresses. Reaction of one of the same fetus, upon the tests, was of "acceleration" type in 46 cases (23\%) (sub-group A-26, sub-group B-20). Estimation according to Apgar scale was 8 - 10 points in 46 cases, newborn period was satisfactory. In 19 cases (9.5\%), reaction in response to the tests was of “deceleration” type (sub-group A-11, sub-group B-8). High estimation according to Apgar scale-8 - 9 points-was registered in 18 fetuses (94.7\%), newborn period was physiological. In one case (5.3\%), estimation according to Apgar scale was 6 - 7 points, the health state of the newborns was estimated as averagely grave.

The "zero" type reaction of the fetus to all three tests was expressed in 23 cases-11.5\% (sub-group A-9, sub-group B-15). In 4 cases out of them (17.4\%), estimation according to Apgar scale was 8 - 9 points, newborn period was physiological. In 19 cases (82.6\%), estimation according to Apgar scale was 6 - 7 points. In early neonatal period, signs of disturbance of adaptation were expressed in 10 cases and in 5 cases, the health state of the newborns was estimated as averagely crave and in 4 cases-as grave.

112 pregnant women, exposed to all three tests in dynamics with one record with one and the same type of the fetus, expressed various types of reaction. In 29 cases (25.9\%), in response to NST, the reaction of the fetus was “deceleration" and in response to the audio stimulus and exercise stress— “acceleration” type. All 29 fetuses had estimation of 8 - 10 points according to Apgar scale and neonatal period was physiological. 14 fetuses (12.5\%), in response to the exercise stress, expressed reaction of type "deceleration" and in response to the audio stimulus and NST_- “acceleration”. Estimation according to Apgar scale in all 14 cases was 8 - 10 points and newborn period was satisfactory. In 69 cases, in response to one of the stresses, the fetuses expressed reaction of type “zero”. In 25 cases (36.2\%), “zero” type reaction was registered in response to the audio stimulus and "acceleration” (17 cases) or "deceleration (8 cases) in response to the exercise stress or NST. In 16 cases (64\%) out of 25, estimation according to Apgar scale was 8 - 10 points and newborn period was physiological. In 9 cases (36\%), estimation according to Apgar scale was 6 - 7 points. The health state of 6 children in early neonatal period was estimated as averagely grave and in one case as grave (died on the 6th day of life); the health state of two children after the proper treatment was improved and they were discharged in satisfactory state. In 8 cases (11.7\%), reaction in response to the audio stimulus and NST was of “acceleration" type (5 cases) or "deceleration” type (3 cases), and in response to the exercise stress- “zero" type. Estimation according to Apgar scale-8 - 10 points - was registered in all 8 cases (100\%) and newborn period was satisfactory. In 11 cases (15.9\%), reaction of the fetus in response to the exercise stress was of “acceleration” type (9 cases) or of "deceleration” type (2 cases), and in response to audio stimulus and NST_- "zero” type. High estimation according to Apgar scale-8 9 points-was registered in two fetuses (18.1\%) and newborn period was physiological. In 9 cases (81.9\%), estimation according to Apgar scale was 6 - 7 points, the health state of the newborns was estimated as averagely grave ( 7 cases) and as grave ( 2 cases).

In 25 cases (36.2\%), reaction of the fetus in response to NST was of "zero" type and in response to the exercise stress and audio stimulus-“acceleration” (19 cases) or “deceleration” (6 cases). Estimation according to Apgar scale-8 - 9 points - was registered in 25 cases. In early neonatal period, three children expressed signs of disturbance of adaptation (reduction of muscular tonus, delayed weight gain); in other 22 children, newborn period was physiological. Index of reactivity of the fetus in response to control tests-exercise stress, audio sti- 


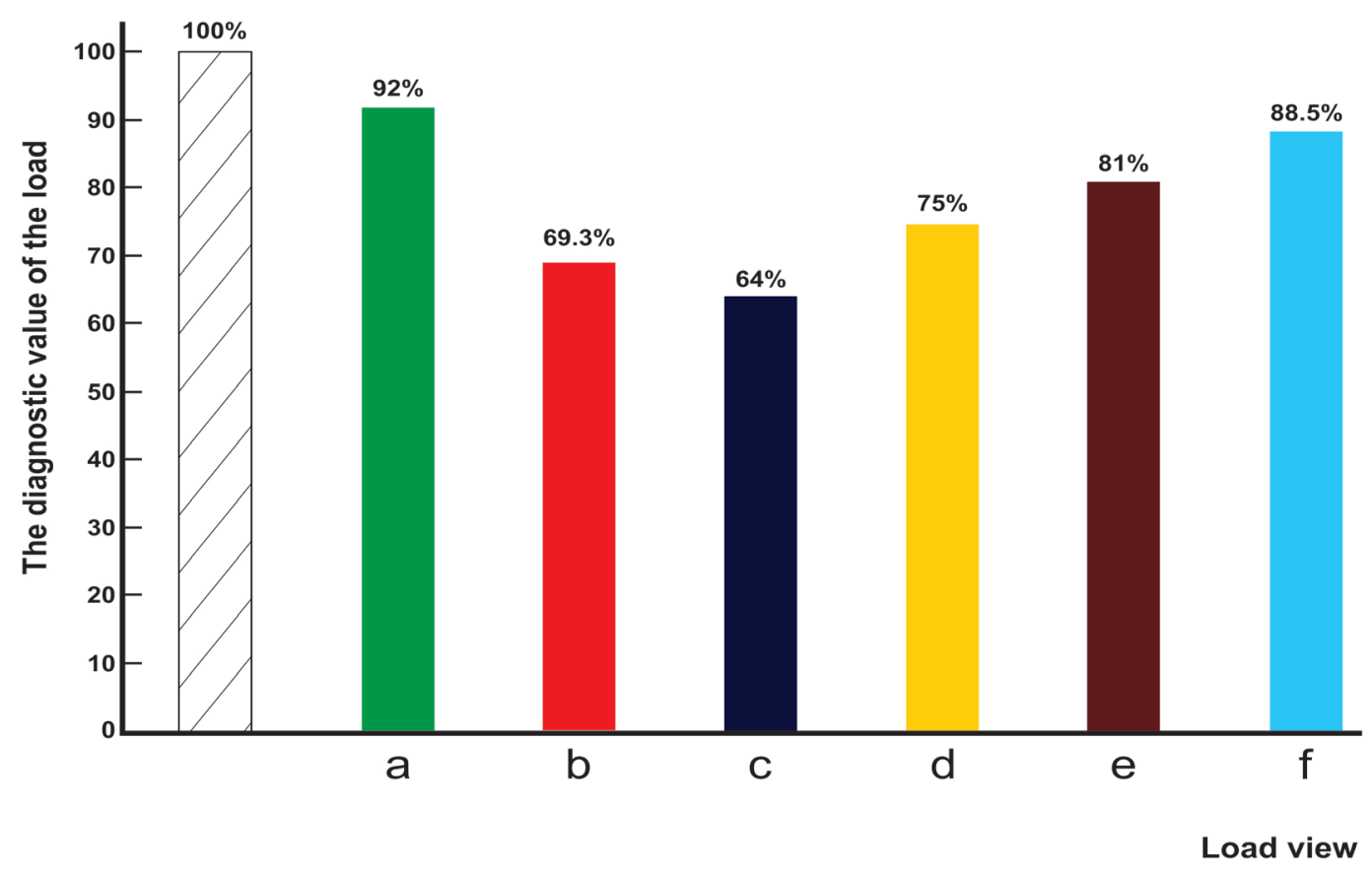

Figure 11. The diagram of the diagnostic value of functional loads at combined application: (a) Exercise stress and audio stimulus; (b) Breath-holding upon exhalation and audio stimulus; (c) Breath-holding upon exhalation and exercise stress; (d) Audio stimulus and NST; (e) NST and Exercise stress; (f) Audio stimulus, exercise stress and NST. On the abscissa - kind of load; on the coordinate-diagnostic value in percent (\%).

mulus and NST—constituted 88.5\%, and diagnostic value constituted $88.5 \%$.

Diagram of comparative diagnostic value of functional tests upon combined application thereof is provided on the (Figure 11).

Hence, on the basis of the analysis of own material, we can conclude that the diagnostic value of the tests upon combined application is significantly increased. The highest diagnostic value was revealed by the set of tests: audio stimulus and exercise stress. The fact is noteworthy that the diagnostic value of all control tests upon combined application of the tests increases compared to isolated application thereof.

\subsection{Definition of the Functional State of the Fetus by Means of Control Tests in "Passive" (Dreamlike State) Hours (IV)}

In view to study characteristics of the reaction of the fetus to the functional stresses, related to the circadian rhythms of the heart activity and motion activity thereof, we have examined 169 fetuses of the III group, subgroup A (with physiological pregnancy), where the reaction of the fetus during the "active" hours (09h00 $14 \mathrm{~h} 00$ and $19 \mathrm{~h} 00$ - 04h00) was of "acceleration" type. Studies have been held during the "passive" (dreamlike state) hours of the fetus from $04 \mathrm{~h} 00$ to $09 \mathrm{~h} 00$ and from $14 \mathrm{~h} 00$ to $19 \mathrm{~h} 00$. We have applied the complex of the tests in capacity of the functional loads: audio stimulus, exercise stress and NST (combined application).

In "passive" (dreamlike state) hours, the background cardio-tachogram of the fetus was expressed with the "flat" type of oscillation. Frequency of the heart rate (FHR) of the fetus fluctuated within $133.6 \pm 1.1$ beats/min. and $138.1 \pm 1.7$ beats/min., constituting averagely $135.8 \pm 1.4$ beats/min. The basal rhythm of the fetus constituted.

We have registered movements of the fetus during the "passive" hours upon external ECG in 7 cases out of $132.5 \pm 1.4$ beats/min. Intra-minute fluctuations constituted $2.5 \pm 0.7$ beats $/$ min. Statistic processing of the data of the background ECG during the "active" and "passive" (dreamlike state) hours of the fetus produced the reliable result according to the following indices: FHR, basal rhythm, intra-minute fluctuations ( $\mathrm{p}<0.5) .169$ (4.1\%) and none of them revealed alteration of frequency of the heart rate of the fetus. Upon functional loads in combined application (audio stimulus, exercise stress and NST) during the passive hours, 155 fetuses out of 169 (91.7\%) expressed "zero" reaction pic.8 The basal rhythm, after functional load, constituted $133 \pm 1.3$ beats/min 
In 14 cases (8.2\%), reaction of the fetus in response to the loads during the "passive" hours (04h00 - 09h00 and $14 \mathrm{~h} 00$ - 19h00) were of "acceleration" type. The latent period constituted $1.2 \pm 0.1 \mathrm{sec}$. Time of achievement of the basal level constituted $31.4 \pm 1.7$ sec. and the level of the basal rhythm after the loads constituted $134.2 \pm 0.8 \%$ beats/min. We have correlated the data of the objective researches on intrauterine state of the fetus with estimation of the newborn according to Apgar scale and the course of newborn period.

164 fetuses out of 169 (95.8\%) that we have studied in dynamics with the "active" and "passive" hours, were born with high estimation according to Apgar scale-8 - 9 points; newborn period of 157 fetuses was physiological. 7 children in early neonatal period expressed the signs of disturbance of adaptation processes. All the children were discharged after 7 - 8 days in satisfactory state.

In 5 cases (2.9\%), estimation according to Apgar scale was lower than 7 - 8 points based on the following reasons: in one case-labor in breech presentation of the fetus aggravated with tilt of hands; in 4 cases-double tight cord entanglement around the neck. The newborn period of one fetus, born with breech presentation, was aggravated with central nervous system damage of light degree; in other cases, the newborn period expressed no aggravations.

Correspondingly, reaction of the fetus during the "passive" (dreamlike) hours in response to the functional tests was absent. On the basis of our studies, we may permit that the "zero" reaction registered during these hours is not the index of unsatisfactory state thereof but indicates to the definite, decreased level of functionality of functional systems of the fetus, related to the dreamlike state thereof (Figure 12).

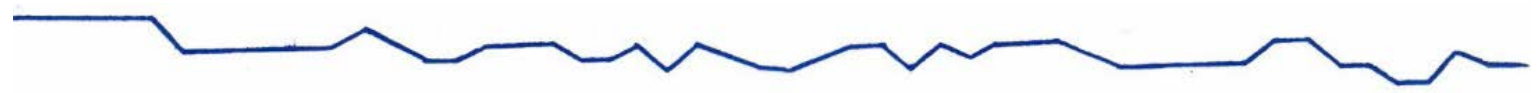

\section{Figure 12. "Zero" type reaction of fetus in response to the physical test.}

Hence, registration of regular alterations of the functional state of physiological systems of the fetus, related to the course of the biological clock, allows differentiation of characteristics of reaction of the fetus to the functional probes, estimating the reserve capacities of organism, developing the criterion of forecasting of the state of the fetus during pregnancy and during labor.

\section{Discussion}

Let's follow the Einstein rule: discuss the problem as simple as possible but no simpler than possible.

Regularities of the rhythmical course of biological processes have long ago started to attract attention of the researchers. Biological rhythms are endogenously generable 24-hour variations of behavior and physiological processes, allowing adapt to the altering conditions of the organisms to environment in the Solar System. Recent studies have revealed the role of melatonin in the sleep-wakefulness rhythm. It has been defined that animals and humans have a hypothalamic "guide" [46], which accents the role of Suprachiasmatic nucleus as of the center, controlling the course of circadian rhythms [47]. Cryptochrome proteins were identified which play their role in generation of daily rhythms etc. [48] [49]. Reaction of organism to external stimulus, at a significant extent, depends on the phase of the rhythmical cycle, in which an organism presents in the moment of study. Significant attention is attached to the selection of the period of time, in which some physiological indices have been defined. Kaar K. [10] and Helbrugge T.H. [15] [16] studied the daily rhythm of FHR of the fetus and a pregnant woman with single auscultation of FHR and the pulse of the mother with the interval of 2 hours during 24 hours. Studies of Konstantinova N.N. [19] and Hoppenbrouwers T. [17] have mainly been aimed at the research of alteration of FHR of the fetus during sleep of the mother. Patrick et al. [50]-[52] studied respiratory movements of the fetus during 1 - 12 hours.

Most of the authors, studying bio-rhythms of various human functions, hold the surveys at 07:00 under the conditions of the main metabolism, at 12:00, when according to the sources, the maximal metabolic activity can be registered, at 17:00 -impact of ingestion and physical activity to the metabolic processes is possible, and at evening hours: 22:00 and 02:00.

Analysis of alteration of HR of a pregnant woman during 24 hours, held with the hereof? methods, revealed that HR of the mother during the daytime averagely constitutes 76.5 beats $/ \mathrm{min}$. and $62.5 \mathrm{beats} / \mathrm{min}$. during the night-time $(\mathrm{p}<0.05)$. Maximal frequency of HR has been registered at 11:00, and the minimal at 04:00—62 
beats/min. ( $\mathrm{p}<0.05)$. Analysis of alteration of FHR of the fetus during 24 hours has revealed that HFR during the daytime averagely constituted 136.5 beats/min. and 132.5 beats/min. during the night hours. The maximal frequency has been registered at 12:00, equaling to 142.5 beats/min., while the minimal—at $06 \mathrm{~h} 00$ (128 beats/min.). Statistic processing of the data on alteration of FHR of the fetus between the day and night hours gave the unreliable result $(\mathrm{p}>0.05)$.

Hence, the conclusion (N1) - analysis of the alteration of frequency of heart rate of the fetus during 24 hours does not give the basis to discuss daily rhythms of heart rates of the fetus (we will be back to this conclusion later).

Methodic approach we applied has a range of privileges: it allows holding/performing/the digital analysis of calculation of the frequency of heart rate of the fetus and the mother according to hourly during 24 hours, we can simultaneously analyze and consider fluctuations and alterations of the levels of functionality of two autonomic nervous systems of the fetus-heart activity and motion activity, uninterrupted recording of FHR of the fetus allowed the application of principally new approach to analysis of daily CTG of the fetus and the mother. The method we applied was complicated, diligent and intensive.

The researcher, studying any of the phenomena, process or subject, aspires to get know as much as possible, to extract as much information as possible. Information reflects real processes, events, peculiarities of the construction. Information exists inasmuch exist material bodies themselves, and correspondingly, heterogeneities created thereby. Every heterogeneity carries some kind of information. For instance, Stars, creating some particular heterogeneity in distribution of matter and energy, serve the source for information. Similar can be stated about any subject or event around us.

Pulse is a heterogeneity for us, and uninterrupted fluctuation of FHR of the fetus (and of the pregnant woman but let's speak about the fetus for now) serves as information for us.

Diagnostics according to the pulse is the greatest art. This method of diagnostics has been applied in China and India for more than five thousand years and is organically related with the traditional Eastern medicine and the representatives thereof on the nature of the disease. The method of diagnostics according to the Pulse is described in the Treaty "Nei Jing", written by the "Yellow Emperor" of China, Huang Di in 1500 B.C. Four thousand years ago, the Chinese doctors, upon diagnosing the disease, attached the greatest importance to study of the pulse and difference of more than 500 types thereof. The ancient Treaty "Huang Di Nei Jing" provided: "distribution of blood to large and small vessels would be impossible without pulsation... it is the pulse that provides blood and pneuma cycles". Hereinafter, the same work indicates to the circular movement of the blood: "Vessels communicate between each other in the cycle". "The hereof cycle has no beginning or end... The blood circulates in the vessels uninterruptedly and circularly..., and the heart is the master over the blood".

Herophilos (340 - 320 B.C.), in his special work "On Pulse”, gave birth to an academic subject/discipline/on arterial pulse. He understood the link between the pulse and the heart activity, defined availability of systole, diastole and intervals in between. Herophilos was the author of the work "Peri sphigmon pragmateias", which was considered as the best Treaty on antiquity and the pulse. Herophilos presumed that the pulse is "motion of the artery" and it allows the identification of "existence of disease in organism and forecasting the future".

Rufus of Ephesus (I-II cent. A.D.) described mechanics of blood circulation, he called the pulse of health people as "eurythmic" proportionality and the pulse of diseased people as "pararhythmic" beside. He described extrasystole, dicrotic and alternating and thready pulse (lat. Pulsus vermicularis) of people in agony. The doctor Aetius, working in Alexandria and Constantinople, described particularities of the pulse in his book "Tetrabiblion" upon anemia, dehydration and malaria. Galen wrote seven books (334 pages) about the pulse, outlining 27 types of pulse, and subdividing each type into three groups. He described sinus and respiratory arrhythmia. The pulse served the basis for Gales to diagnose stomach diseases of the Emperor Marcus Aurelius. Ancient doctors perfected the method of diagnostics according to the pulse [53].

The short tour to the past (the greatest past) proved importance of the Pulse. And if hundreds of researches have been held to define the daily rhythm of the Pulse of heart activity of a grown-up human (we have defined the daily rhythm of a pregnant woman), then the researches on biorhythms of the intrauterine fetus are singular (4 - 5 articles) and contradictory, bringing no information. The heartbeat of the fetus is the basic and important (if not the most important index) of functional state of the fetus and vital activity thereof. Ancient doctors perfected the method of diagnostics according to the pulse of a grown-up man and correspondingly... we go back to the analysis of the CTG of the fetus.

We, exposing the daily CTG of the fetus to the visual analysis, have attached our attention to the heterogenei- 
ty of structures thereof, which allowed us to define four types of oscillation, registered on the hourly CTG in various combinations and sequence, uninterruptedly interchanging. Each of the types of oscillation, so different according to the form, amplitude and duration, was respectively named as: "pointed", "round", "flat” and "mixed".

Partitioning the daily and hourly CTG (for each pregnant woman 24 tachograms were composed of the indices of maternal and fetal heartbeat), exposing it to a scrupulous visual and digital analysis, we have clearly registered the irregularity of distribution of one or another type of oscillation on the daily rhythmogram. Calculating the quantity of time, during which each of the CTG types is repeated during one hour of the survey, we have attached our attention to the fact that the period of time has been twice registered during 24 hours, when the background CTG of the fetus was of "flat" (III) type of oscillation (52\% of registration time) prevailing over other types (I, II, IV) of oscillation. We called this time—-04:00 - 09:00—and-14:00 - 19:00—as “passive” hours (or "dreamlike" state of the fetus). During the rest of the time, from 09:00 to 14:00, and from 19:00 to 04:00, the background CTG of the fetus was registered with the "mixed" type of oscillation, on the background of which types I and II of oscillation were recorded (87.8\% of recording time). We called these periods as "active” hours.

The daily ECG of a pregnant woman also were exposed to the analysis: we carried out the calculation of the recording time of "flat" type of oscillation on CTG according to hours. As we found out, the duration of the "flat" (III) type of oscillation during sleep exceeded duration of the rest (I, II < IV) of the types. During the day, within pregnancy, the "flat" type of CTG was either absent or recorded on the hourly CTG during the short period of time (2 - 5 minutes).

The analysis of motion activity of the fetus during 24 hours revealed that the duration of motion during the "passive” hours for one hour of recording exceeded $1 \mathrm{~min} .50 \mathrm{sec}$., and for the whole period of "passive" hours, constituted $11 \mathrm{~min}$. $5 \mathrm{sec}$, which corresponds to 2.6\%. In "active” hours, motion of the fetus for 1 hour of recording constituted 3 and over minutes, achieving the maximal value of 32 min. \pm 0.2 within 22:00 - 23:00. Motion of the fetus during the whole period of “active” hours was recorded during 91 min. 10 sec., which constituted $16 \%$ of the total time. Calculation of the number of heart rates of the fetus lower than 120 beats/min and higher than 150 beats/min (registered on each TG during 24 hours) revealed the maximal number of indices of FHR lower than 120 beats/min: the first at 06:00 - 07:00, the second at 15:00 - 19:00, with higher expression at 17:00 - 18:00. FHR higher than 150 beats/min. was as well registered in capacity of two peaks: the first at 11:00 - 12:00, and the second at 19:00 - 01:00 (evening-night hours).

Preliminary analysis: four types of oscillation have been expressed on the daily CTG of the mother and the fetus in physiological pregnancy: "pointed”, “round”, “flat”, and “mixed” types. Clearly expressed daily rhythm of the heart activity was maintained during pregnancy of the mother. The minimal FHR was recorded during the night hours (during sleep) from 02:00 to 04:00, and the maximal—at 12:00 - 13:00 (active vigilance).

"Passive" hours (04:00 - 09:00 and 14:00 - 19:00) and "active" hours (09:00 - 14:00 and 19:00 - 04:00) have been expressed depending on prevalence of one or another type of CTG upon recording of the daily EEG of the fetus, which served the basis to conclude that during 24 hours, the increase and decrease periods of physiological functions of an organism inter-change for the fetus, as of a adult. According to our data, "active" hours of the fetus coincide with the "passive" hours of the mother, and "passive" hours of the fetus coincide with "active" hours of the mother.

Studies of the daily rhythm of motion activity of the fetus indicate to an active state of the fetus upon decreased physiological function of the mother. Maximal number of motion of the fetus is recorded from 22:00 to 04:00. At the same time, high activity of the fetus had no impact on the night sleep course of a pregnant woman.

During the "passive" (or "dreamlike") hours of the fetus from 04:00 to 09:00, motion activity thereof was reduced 6 - 7 times (2 - 3 minutes per 1 registration hour).

The result of calculation of FHR lower than 120 beats/min. and higher than 150 beats/min. is of interest. Similarly to an adult, the maximal number of indices of FHR of the fetus lower than 120 beats/min. is revealed during the "passive” (or “dreamlike”) hours, while frequencies higher than 150 beats/min. dominate during “active" hours of the fetus.

\section{Conclusions}

We have defined the daily rhythms of intrauterine fetus. The level of functionality of physiological systems of the fetus is in concord with the systems of the organism of the mother, but is in the reverse phase.

The data obtained allowed us to revise of our initial conclusion (N1) and unifying the results of both studies, 
to suggest the following conclusion: we can presume that a fetus, during the intrauterine development, needs comparatively constant homeostasis, which can be achieved by the activation of the fetus during "passive" hours of the mother and via the "decrease" of physiological functions of the fetus (better state: moves to the dreamlike state) upon the increase of those of the pregnant woman.

The results we obtained from scientific research have been immediately inculcated in the Clinic (see the material on studied fetuses in the Chapter "Contents"). By means of external cardiography using the tests, we have studied the peculiarities of the functional state of the fetus, taking into account the biologic rhythms thereof we defined. We have carried out studies during the "active" and "passive" hours (or "dreamlike" state) of the fetus. Using the functional stresses, we have obtained three types of reaction- "acceleration", "deceleration" and "zero".

In the group of the fetuses with the reaction to the functional loads (see in the Chapter "Definition of the Functional State of the Fetus by means of Control Tests in "active" and "passive" (or "dreamlike") hours) according to the "acceleration" type, $94.5 \%$ high estimation according to Apgar scale ( 8 - 10) points has been registered. Latent period constituted $2 \pm 0.2 \mathrm{sec}$. (sub-group A), $1.9 \pm 0.7 \mathrm{sec}$. (sub-group B). Restoration time of FHR of the fetus to the initial point constituted $35.0 \pm 2.6$ sec. in the sub-group A and $39 \pm 3.1$ sec. in the sub-group B.

With the reaction of "deceleration" type, in 76\% cases, estimation according to Apgar scale was 8 - 10 points and in $24 \%$ - estimation was lower than 8 points. Latent period constituted $1.3 \pm 0.4 \mathrm{sec}$. in sub-group A and 1.9 \pm 0.9 sec. in sub-group B. Restoration time of FHR of the fetus to the initial point constituted $37.0 \pm 3.4 \mathrm{sec}$. in the sub-group A and $39.0 \pm 3.8 \mathrm{sec}$. in the sub-group B.

In the group of the fetuses with "zero" type of reaction to the stress with high estimation according to Apgar scale (8 - 10 points), $41.2 \%$ of fetuses were born. In $58.8 \%$ cases, estimation according to Apgar scale was lower than 8 points ( 3 - 7 points). Intrauterine death of the fetus was registered in 7 cases and in 5 cases with estimation according to Apgar scale lower than 8 points (3 - 7 points), 37.2\% of newborns had various disorders of early neonatal period.

According to various authors, acceleration of FHR of the fetus in response to the load is the index of satisfactory state thereof. Demidov V.N. and etc. [11], Pereira-Lur N. [42] and others argue that acceleration of FHR of the fetus indicates disorder of the intrauterine state and adaptive capacities thereof.

According to the data of the studies held by Fischer W.M. [54], Freeman R.K. and etc. [55], Patrick J. and etc. [50]-[52] a stimulus for various objects of study entails various reactions according to the features of the nervous system, as well as according to the functional state of organism during the study. Correspondingly, various types of reaction of the fetus to the stress can be registered with various levels of maturity of sympathetic and parasympathetic nervous system; it may depend on the functional system of an organism of the mother and the fetus during the study.

On the basis of our data, we can conclude that a healthy fetus, in "active" hours, responds to the functional load with acceleration or deceleration of FHR with short latent period, fast recovery to the initial level and maintenance of the basal rhythm after the test(s).

The best reaction of the fetus is the "acceleration" type. Light extension of latent period of the fetuses attributed to the sub-group B compared to the fetuses in the sub-group A is interesting, as it indicates to the negative impact of pathologic pregnancy to the fetus and can be used as the earliest sign of disorders in the state of the fetus.

Particular attention shall be attached to the fetuses with stable rhythm, that is "zero" type of reaction to the load. This type of reaction, registered in "active" hours, indicates to decreased compensative mechanisms of the fetus and is, as we presume, the index of the transitional state from healthy to pre-pathologic.

According to D. Navot [41] [43], Pereira-Lur N. et al. [42] absence of the reaction of the fetus upon the testsshall be estimated as pathologic condition, evidencing intrauterine hypoxia of the fetus.

Studying the reactivity of the fetus to the control tests and holding the comparative characteristics thereof, we have concluded that upon the isolated application of the functional loads, the exercise stress and audio stimulus (68.5\%) give the optimal information, while NST-58.7\%. Upon combined application of the tests, value thereof achieves $92.3 \%$.

Hence, application of two and over diagnostic tests in "active” hours in view of definition of intrauterine state of the fetus during pregnancy, significantly increases diagnostic value of external ECG of the fetus, which in its turn, allows estimating compensating-adaptive mechanisms of the fetus with high accuracy and facilitates cor- 
rect orientation of the clinicians.

In "passive" (dreamlike) hours, the background CGT of the fetus was expressed by the "flat" type of oscillation. Frequency of heart rates fluctuated within $133.6 \pm 1.1$ beats/min. to $138.1 \pm 1.7$ beats/min, averagely$135.8 \pm 1.4$ beats $/ \mathrm{min}$. The basal rhythm was $132.5 \pm 1.4$ beats $/ \mathrm{min}$. Intra minute fluctuations constituted $2.5 \pm$ 0.7 beats/min. Movements of the fetus were registered in $4.1 \%$ cases.

Upon application of the tests during "passive" hours, 91\% expressed "zero" type of reaction, however, only in 2.9\% the fetuses were born with estimation according to Apgar scale lower than 8 points (6 - 7 points). In other 97.1\%, estimation according to Apgar scale was 8 - 10 points, and newborn period of fetuses was physiological.

Correspondingly, in "passive" hours, the reaction of the fetus to functional loads is mostly absent. On the basis of the results of own studies, we can argue that "zero" type of reaction of the fetus during these hours does not indicate to an unsatisfactory state but rather to a decreased level of functionality of physiological systems of the fetus, related to dreamlike state. "Zero" type of reaction, accepted by many of the researches and inculcated in many clinics, deserves attention upon registration thereof in "active" hours of the fetus.

Thus, the account of regular alterations of the functional state of physiological systems of the fetus, related to the biological clock thereof, allows the differentiation of peculiarities of the reaction of the fetus to the functional tests and enables us to estimate the reserve capacities of the organism to develop the criterion of forecasting of the state of the fetus during pregnancy and delivery.

As mentioned above, based on the results of own studies, widely applied in the clinic (more than 100 thousand pregnant women), we have significantly extended diagnostic capacities of CTG of the fetus, and even further, we have provided high percentage of accurate estimation of functional state of the fetus during pregnancy, and developed the program of forecasting. Studying characteristics of pulse wave, it becomes perfectly clear that the definition of the state of not only internal organs but also of the whole organism of intrauterine fetus is possible.

Studies that we performed are the scanty part of evolution in diagnostics of the intrauterine state of the fetus. Study of the bio-rhythms of the fetus showed us the rhythms of the nature [6] [8].

The basic rhythms of Nature that left their imprint on the existence of all living organism on Earth, arose under the influence of the Earth's rotation relative to the Sun, the Moon and other planets and stars of the Universe. This periodicity gave rise to the rhythm that has become essential for their life. Life is a continual chemical process of building up and breaking down of organic substances, that results from the substance exchange between an organism and the environment. This makes it impossible for a living organism to exist without the external environment.

Based on the above, it seems very reasonable to put a question: "Does the human fetus really develop within the uterus without experiencing any external or cosmic influence?” The period of human intrauterine development lasts for nine terrestrial months (or ten lunar months, i.e. 40 weeks). Within this term a tremendous evolutionary breakthrough takes place when a living being completely forms from two solitary cells, with fully fledged internal organs. This creature starts living and developing in the external world. At what point an absolute potential transforms into a human being? What are the interrelation mechanisms of biological clock between a pregnant woman and the fetus and their synchronization with the environment and Cosmos? [56].

What Cosmos is? Is it the Universe? Solution of this issue is the key to solution of all other issues, including the issues related to the problem of cosmic impact on human life. Attempts to clarify one or another problem, regarding the development of intrauterine fetus from conception to delivery, lead to unsolved mysteries again and again.

Life starts from one cell. In two days after conception, number of cells increases twice. On the $4^{\text {th }}$ week of pregnancy, the fetus is no larger than a kernel of rice with the body being already formed. Heart and spine, legs and hands start to appear. On the $8^{\text {th }}$ week, the fetus is like a nut and main organs start to develop: brain, liver, lungs, bones, sense of touch. The fetus starts an impetuous growth. On the $26^{\text {th }}$ week, the fetus starts to taste, responds to light and sound and can clench his hands. And all of this is generated from one cell! How such speed is possible?! The assemblage of a normal human takes 9 Earth and 10 Moon months (40 weeks of pregnancy). It is too fast.

We would like to dedicate some time to the natural Earth satellite-Moon-non-luminous heavenly body, reflecting the solar light. Moon is exposed to the Earth always with one side. The Lunar rhythm is a repeating alteration of the intensity and character of biological processes. There are lunar-monthly (29.4 days and nights) and lunar-daily (24.8 hours) rhythms. Many plants and animals, as well as many sea animals and seaweeds re- 
produce in a particular phase of the lunar cycle. Moon participates in vital activity of a human, in the process of conception and the course of pregnancy, which prompted us to add graphics- "Biorhythms Moon" to our "QUESTIONNAIRE SLEEP-WAKE CYCLE OF A PREGNANT WOMEN".

In considering the construction of an organism we have come to the idea this order, stability, accurate distribution and functioning of all systems, could not be formed by themselves spontaneously. Life starts with conception. At the very first minutes of conception, when the future parents do not even suspect of possible pregnancy, all the systems of a female organism, particularly the whole hormonal system switches to the new level of order: epiphysis is increased, corticotropine controls hormones of adrenal cortex, synthesis of progesterone, androgens and oestrogens is enhanced, many other processes in various cells are regulated, for instance in osteoblasts, responsible for the formation of bones, which is necessary for the fetus from the very first week.

The great breakthrough in the science allowed witnessing the process of development of a fertilized ovum?, the sizes of which are too small. Considering the development of the embryo with the comparison of emergence of the Universe, we can find lots of amazing coincidences. Big bang, leading to the formation of the new Universe, reminds of the extremely fast pace of development of the embryo on early stages. Transmission of information, contained in DNA to the matter of a living organism leads to the enlargement of an embryo 50 times during one month only, and in fact it is like the event, occurring in Cosmos. However, many astrophysicists adhere to the opinion that the process of formation of the Universe (unlike the regular development of an embryo) - is uncontrollable. Nevertheless, modern astronomic observations allowed the scientists to come to the conclusion that the supercluster of galaxies is nothing but cellular constructions. The sizes only of such "cells" are far disproportionate compared to human cells. The recent discoveries speak in favor of this theory. Thus, Cosmic telescope Spitzer allowed discovering the Stellar system with the length of 80 light years, resembling a twisted DNA chain. In the moment when the spermatozoon penetrates the ovum, the micro explosion takes place and the information about future personality "bursts out". This phenomenon can be compared with the Big Bang and the birth of a new Star or Universe.

The most famous formula of Hermes states: "Whatever is below is similar to that which is above. Through this the marvels of the work of one thing are procured and perfected". We apologize to the Great Hermes that we afford? to accomplish the hereby studies inasmuch as after Your formula, we have nothing to say...

However, let us accomplish the publication with the address to the astronauts and astrophysicists: let us join Your Scientific Researches since physiological aspects of the state of an organism are better to be studied jointly with doctors (particularly, obstetrician-gynecologists), biologists and physiologists. The present study has been the first in the world's medicine and bio-rhythmnology to detect and establish the daily rhythms of cardiac and motor activity in the human fetus. Initially, the results were presented in February, 1980 at the session of Academic Council of the Institute of Perinatology, Obstetrics and Gynecology (at present-K.V. Chachava Institute of Perinatology, Obstetrics and Gynecology) being then immediately implemented into the Institute's clinical practice. Thus we confirmed the importance of external EKG and further CTG in the assessment of the intrauterine fetal condition. Successfully combining my specialty as gynecologist-obstetrician with functional diagnostics of fetal health for more than 30 years, I have never found any incorrect diagnostic findings, which strengthens my confidence that our study will rightly prove the high diagnostic value of fetal and maternal biorhythmicity.

\section{Acknowledgements}

My particular gratitude goes to M. Devdariani, MD, PhD in Pediatric Neurology, Head of the Laboratory of Electrophysiology; M. Kokhia, biologist, $\mathrm{PhD}$ in Biology and A. Shanava, nurse.

\section{References}

[1] Dedov, I.I., et al. (1992) Biorhythms Hormones. M. Medicine, 256.

[2] Halberg, F., Visscher, M.B., Flink, E.B., Berge, K. and Bock, F. (1951) Diurnal Rhythmic Changes in Blood Eosinophil Levels in Health and in Certain Diseases. The Lancet, 71, 312-319.

[3] Johansson, B., Wedenberg, E. and Westin, B. (1964) Measurement of Tone Response by the Human Foetus. A Preliminary Report. Acta Oto-Laryngologica, 57, 188-192. http://dx.doi.org/10.3109/00016486409136960

[4] Klein, D., et al. (1978) The Hypothalamus. Raven Press, New York, 303-327.

[5] Savitsky, G.A. (1964) Peculiarities of Fetal Response to Functional Loading Associated with Its Biological Clock. Fet- 
al Response to Insulin Injected into Diabetic Mother as a Clinical Functional Test. In: Fetal and Neonatal Hypoxia, 103-108.

[6] Alyakrinsky, B.S. (1967) Biological Rhythms in Space. Proceedings of Studies Dedicated to K.E. Tsiolkovski’s Scientific Heritage and Development of His Ideas, Kaluga, Section: Problems of Space Biology and Medicine, 49-58.

[7] Alleyne, G. and Young, V.H. (1967) Adrenocortical Function in Children with Severe Protein-Calorie Malnutrition. Clinical Science, 33, 189-200.

[8] Aschoff, I. (1971) Cirkadian Rhythmus in Space Medicine. Man in Space. Proceedings of the 4th International Symposium on Basic Problems of Human Life in Space, Erevan, 1-6 October 1971, 264-284.

[9] Harbert, G.M., Coiner, I.M., Turner, S.M. and Atuk, N.O. (1981) Biorhythms of the Nonpregnant Human Uterus. The Journal of Reproductive Medicine, 26, 493-498.

[10] Kaar, K. (1980) Antepartal Cardiotocography in the Assessment of Fetal Outcome. Acta Obstetricia et Gynecologica Scandinavica, 94, 1-56.

[11] Demidov, V.N., et al. (2005) Antenatal Cardiotocography. Health and Medicina, No. 9, 52-55.

[12] Hon, E.H. (1961) The Electronic Evolution of Fetal Heart Rate. Y. The Vagal Factor in Fetal Bradycardia. American Journal of Obstetrics \& Gynecology, 82, 291-300. http://dx.doi.org/10.1016/0002-9378(61)90061-8

[13] Kudli, F. (1971) Iatrogenic Fetal Hypoxia. In: Gevers, K.H. and Ruys, J.H., Eds., Physiology and Pathology in the Perinatal Period, Springer, Dordrecht, 57-75.

[14] Schifrin, B.S., et al. (1974) Evaluation of Oxytocin Challenge Test. Obstetrics \& Gynecology, 43-617.

[15] Hellbrugge, Th. (1959) Schlafen und Wachen in der Kindlichen Entwicklung. Beiheftezum Arch. Kinders 39, Enke Verlag, Stuttgart.

[16] Hellbrugge, Th. (1964) Development of Circadian Rhythms in Child. The Biological Clock. Collected Articles. Mir, Moscow, 510-530.

[17] Hoppenbrouwers, T., Combs, D., Ugartechea, J.C., Hodgman, J., Sterman, M.B. and Harper, R.M. (1981) Fetal Heart Rates during Maternal Wakefulness and Sleep. Obstetrics \& Gynecology, 57, 301-309.

[18] Arkhangelskaya, N.A. (1954) Topography of Skin Temperature and Daily Periodicity of Body and Skin Temperature in Newborns. In: Experience in the Study of the Physiological Function Regulation, USSR Academy of Sciences, 177-184.

[19] Konstantinova, N.N. (1967) Disturbance of Placental Circulation and Cardiac Performance of the Fetus (Pathogenesis and Theoretical Prerequisites to Early Diagnosis and Treatment). Abstract, Thesis for Doctor's Degree in Medical Sciences, The Research Institute of Obstetrics, Gynecology and Reproductology, Leningrad, 27 p.

[20] Kintraia, P.Y., Zarnadze, M.G. and Bogotany, S. (1993) To the Question of Study of Some Circadian Rhythms in Pregnant and Fetus (Gestation Age of 16 - 30 Weeks). Recent Progress in Perinatal Medicine VIII. Edited by Gati, I., Budapest, 34-35.

[21] Kintraia, P.Y. and Zarnadze, M.G. (2000) Interdependence of Circadian Rhythms of Pregnant and Fetus of 16 - 40 Weeks of Gestation. 16th FIGO World Congress of Gynecology and Obstetrics, Washington DC, 3-8 September 2000, 107.

[22] Kintraia, P.Y., Zarnadze, M.G., Kintraia, N.P. and Kashakashvili, I.G. (2004) Daily Rhythms of the Human Fetus, Their Interdependence and Concordance with Mother’s Biorhythms. Bulletin of Russian Academy of Natural Science, 4, 70-76.

[23] Kintraia, P.I., Zarnagze, M.G., Kintraia, N.P. and Kashakashvili, I.G. (2005) Development of Daily Rhythmicity in Heart Rate and Locomotor Activity in the Human Fetus. Journal of Circadian Rhythms, 3, 5.

[24] Zarnadze, M.G. (1977) Changes of Fetal Heart Rate in Relation to Circadian Rhythms of Pregnant Women. Medical Scientific Conference of Young Physicians of Heorgic, Bakuriani, 9-12 April 1977, 419-420.

[25] Zarnadze, M.G. and Devdariani, M.G. (1979) Evaluation of the Functional State of the Fetus during Pregnancy by Method of Abdominal Electrocardiography. Tracking the Diminution of Perinatal Pathology. Collected Articles. Tbilisi, 1, 162-167.

[26] Zarnadze, M.G. (1982) Peculiarities of Fetal Response to Functional Loading Associated with Its Biological Clock. Tbilisi, 15-20.

[27] Zarnadze, M.G. and Devdariani, M.G. (1983) Adaptive Re-Adjustment of Fetal Cardiac Biorhythms during Physiologic Pregnancy. 14th Congress of Obstetrics and Gynecology: Problems of Perinatology. Diagnosis and Treatment of Fetal Infertility, Kishinev, Abstracts, 172.

[28] Zarnadze, M.G. (1983) Diagnosis of the Functional State of the Fetus by Means of the Use of Outer Electrocardiography Functional Loads and Subject to a Daily Frequency Rhythms of the Fetus. Academician Chachava Scientific Re- 
search Institute of Perinatal Medicine, Obstetrics and Gynecology. Guidelines. Tbilisi, 28.

[29] Zarnadze, M.G. (1985) 24-Hour Periodicity of Fetal Heartbeat and Locomotor Activity as the Indicator of Fetal Functional State during Pregnancy. Abstract, Thesis for Candidate’s Degree in Medical Science, Banner of Labir Research Institute of Pediat, Kiev, 34.

[30] Zarnadze, M.G. (1989) Peculiarities of Fetal Response to Functional Load Associated with Its Biological Clock. Collected Articles of the RI PMOG, MHC GSSR, 15-20.

[31] Zarnadze, M.G. (1990) Fetal Reactivity yo Functional Load. Materials of the 3rd Congress of Obstetrics and Gynecology of Georgia, Tbilisi, 13-15 November 1990, 18.

[32] Zarnadze, M.G. and Kintraia, N.P. (2002) Circadian Rhythms of the Fetus at 16-28 Weeks' Gestation. Journal of Obstetrics \& Gynecology, Moscow, 6, 58-59.

[33] Zarnadze, M.G., Kintraia, N.P. and Kvavadze, I. (2003) Biological Clock of the Human Fetus. 7th International Symposium on Intrauterine Surveillance RCOG, London, 9-11 June 2003, 60.

[34] Zarnadze, M.G. (2003) Maturity Degree of Endogenous Pacemaker System of Regulation of Human Fetus Circadian Rhythms. 7th International Symposium on Intrauterine Surveillance RCOG, London, 9-11 June 2003, 61.

[35] Zarnadze, M.G. (2003) External and Internal Developmental Aspects of Circadian Rhythms in the Human Fetus. The 3rd Congress of Azerbaijan Obstetrics and Gynecology, Baku, 310-316.

[36] Zarnadze, M.G. (2006) Circadian Rhythms of Human Fetus and Their Coordination with Its Functioning. Georgian Medical News, Tbilisi-New York, No. 9, 59-62.

[37] Alekperov, M.A. (1965) Daily Fluctuations of the Functional State of the Adrenal Cortex in Hypertension. Azerbaijan Medical Journal, No. 1, 27-32.

[38] Baluda, V.P., et al. (1978) Biological Rhythms System of Human Homeostasis. Frunse, Ilim, 50-83.

[39] Challis, J.R.G. and Patrick J.E. (1983) Changes in the Diurnal Rhythms of Plasma Cortisol in Women during the Third Trimester of Pregnancy. Gynecologic and Obstetric Investigation, 16, 27-32. http://dx.doi.org/10.1159/000299209

[40] Claus-Walkor, J. (1969) Daily Rhythms of Electrolytes and Aldosterone Excretion in Men with Cervical Spinal Cord Section. The Journal of Clinical Endocrinology \& Metabolism, 29, 300-301. http://dx.doi.org/10.1210/jcem-29-2-300

[41] Navot, D., Yaffe, H. and Sadovsky, E. (1983) Diagnosis of Fetal Assessment of Fetal Movement and Heart Rate Accelerations. Journal of Perinatal Medicine, 11, 175-178.

[42] Pereira-Lur, N. (1982) Respuesta auditiva provocada: Nuevo metodo de evaluacion fetal. Revue Francaise de Gynecologie et d Obstetrique, 41, 386-400.

[43] Navot, D., Donchin, Y. and Sadovsky, E. (1982) Fetal Response to Voluntary Maternal Hyperventilation. A Preliminary Report. Acta Obstetricia et Gynecologica Scandinavica, 61, 205-208. http://dx.doi.org/10.3109/00016348209156557

[44] Kochia, M.I. (1980) Electrophysiological Parameters of the Fetus and Their Changes at Functional Loading during Labor. Abstract of the Thesis for Ph.D. Degree in Biology, Institute of Physiology, Tbilisi, 26 p.

[45] Stembera, Z.K., et al. (1970) Simplified Step Test for the Diagnosis of Antenatal Threat to the Fetus by Hypoxia. Ceská Gynekologie, 35, 169-171.

[46] Nakamura, K. (2010) Review of Particle Physics. Journal of Physics G: Nuclear and Particle Physics, 37, 075021. http://dx.doi.org/10.1088/0954-3899/37/7A/075021

[47] Bernstein, J. (2014) A Chorus of Bells and Other Scientific Inquiries. Word Scientific Publishing Company, Singapore, 200 p. http://dx.doi.org/10.1142/9046

[48] Jedicke, R., Granvik, M., Micheli, M., Ryan, E., Spahr, T. and Yeomans, D.K. (2015) Surveys, Astrometric Follow-Up and Population Statistics. [arXiv:1503.04272v1]

[49] Steven, R., et al. (2015) The Apache Point Observatory Galactic Evolution Experiment (APOGEE). Submitted to the Astronomical Journal: 50 Pages, Including 38 Figures, 4 Tables, and 5 Appendices. [arXiv:1509.05420v1]

[50] Patrick, J. (1978) Human Fetal Breathing Movements and Grass Fetal Body Movements at Weeks 34 to 35 of Gestation. American Journal of Obstetrics \& Gynecology, 130, 693-699. http://dx.doi.org/10.1016/0002-9378(78)90330-7

[51] Patrick, J., Campbell, K., Carmichael, L., Natale, R. and Richardson, B. (1982) Patterns of Gross Fetal Body Movements over 24-Hour Observation Intervals during the Last 10 Weeks of Pregnancy. American Journal of Obstetrics and Gynecology, 142, 363-371.

[52] Patrick, J., Campbell, K., Carmichael, L. and Probert, C. (1982) Influence of Maternal Heart Rate Gross Fetal Body Movements on the Daily Pattern of Fetal Heart Rate near Term. American Journal of Obstetrics \& Gynecology, 144, 533-538. http://dx.doi.org/10.1016/0002-9378(82)90222-8 
[53] Michael, S. (2005) 100 Great Doctors. 528.

[54] Fischer, W.M. (1973) Grundlagen und klinische Wentigkeit der Kardiotokographie. In: Fischer, W.M., Ed., Kardiotokographie, Lehrbuch und Atlas, Georg Thieme Verlag, Stuttgart, 43-179.

[55] Freeman, R.K., Garite, T.J., Mondanlou, H., Dorchester, W., Rommal, C. and Devaney, M. (1981) Postdate Pregnancy: Utilization of Contraction Stress Testing for Primary Fetal Surveillance. American Journal of Obstetrics \& Gynecology, 140, 128-135. http://dx.doi.org/10.1016/0002-9378(81)90099-5

[56] Zarnadze, M. and Ninashvili, N. (2012) Biologo-Astronomic Principles of Daily Rhythms Self-Regulation in Human Fetus. International Journal of Gynecology and Obstetrics, 119, S732. http://dx.doi.org/10.1016/s0020-7292(12)61809-8

\section{List of Abbreviations}

bpm: beats per minute

CTG: cardiotachogram

ECG: electrocardiogram

FHR: fetal heart rate

h: hour(s)

HR: heart rate

NST: non-stress test

Sek: second(s) 


\section{Questionnaire Sleep-Wake Cycle of Pregnant Women}

Date of examination

Number of medical history

Surname, first name, patronymic

Age, (date of birth)

Domestic living conditions

Weight: before pregnancy

Occupation during pregnancy

Unhealthy occupational factors (dust, noise, increased gas levels, increased humidity etc)

Duration of working hours

before pregnancy

during pregnancy

How much important is sport in your life (What kind of sports have you played? How long? (age)

before pregnancy

during pregnancy

Your leisure time (What do you do in your free time?)

before pregnancy

during pregnancy

This pregnancy is desirable for either spouse

This pregnancy is not desirable

for either spouse

for one of them

Are you satisfied with the quality of your night-time sleep before pregnancy?

Did you have any sleep problems? Which exactly?

How long did these problems last? -

Unhealthy habits (alcohol, cigarettes, drugs)

before pregnancy

during pregnancy

Do you drink coffee, tea? How much?

before pregnancy

during pregnancy

What time do you go to bed (exact time)?

before pregnancy

during pregnancy

How long does it take you to fall asleep (or indicate the time of quiet waking)

before pregnancy

during pregnancy

What time do you wake up in the morning?

before pregnancy

during pregnancy

How often do you wake up during the night?

before pregnancy

during pregnancy

Cause of night-time wake-up during pregnancy

urination

fetal movements

other 
Total duration of night-time waking during pregnancy (minutes, hours)

What influences the quality of your night-time sleep (color of the surrounding setting the bed, the bedroom, smells etc.)

Do you feel relaxed when you wake-up in the morning?

before pregnancy

during pregnancy

Do you go to bed in the day-time

Average duration of day-time sleep during pregnancy

Do you feel drowsy in the day-time? What do you think it is associated with?

before pregnancy

during pregnancy

The period of the highest levels of efficacy

before pregnancy

during pregnancy

Have you ever had a feeling of decrease in physical and mental activity (indicate exact time if the answer is positive)

Have you ever experienced anxiety, depression, irritation (duration and period of the day)?

Do you have periods of tiredness, fatigue during the day?

Do you have memory problems?

before pregnancy

during pregnancy

During which period of the day is it especially difficult for you to concentrate?

before pregnancy

during pregnancy

Which somatic diseases did you have before pregnancy

Which somatic diseases have been revealed in pregnancy

Are you allergic (if yes, since when (age)?

Diseases of the thyroid gland

Number of operations due to termination of pregnancy induced abortion

spontaneous abortion

mini-abortion

Did you have any post operative complications?

Changes in night-time sleep quality, if these took place after operations, narcosis etc.

Did you undergo medicamentous treatment during pregnancy (if yes, what kind of treatment?

Biorhythms Moon

\section{Moon Phase}

Tides and low tide

Do you use sedatives? Which?

before pregnancy

during pregnancy

Comment of the pregnant on her night-time sleep (snoring, bruxizm, respiratory arrest (apnea), dispnea, cramps in lower extremities etc.) 
Since when (term of pregnancy) have you had sleep disorders?

Which of the positions do you take during sleep?

any position

supine position

lateral position

Gravida (number of pregnancies)

Is this your second (third, fourth etc) pregnancy? If yes, did the quality of your night-time sleep change from pregnancy to pregnancy? (Describe these changes)

\section{Conclusion:}

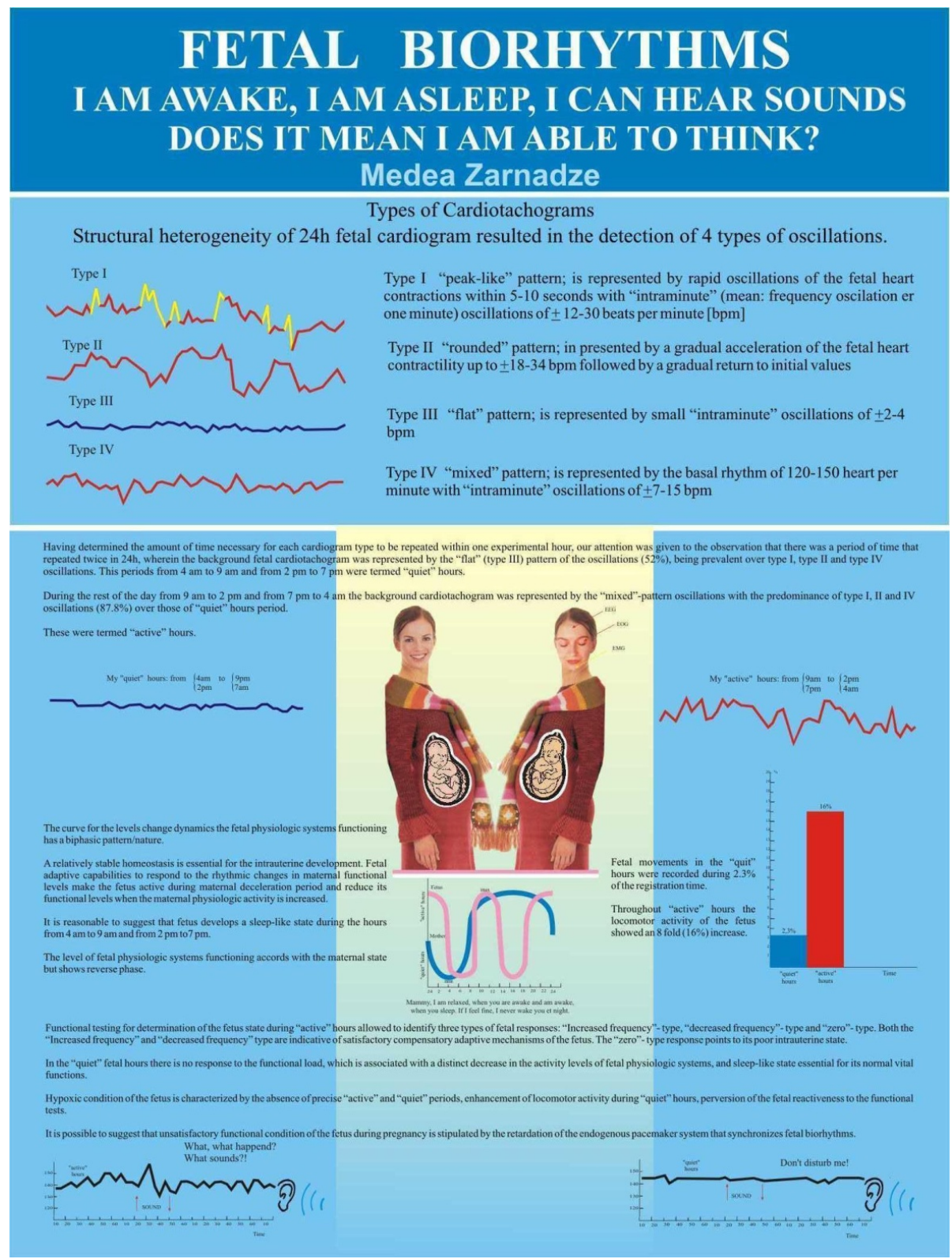




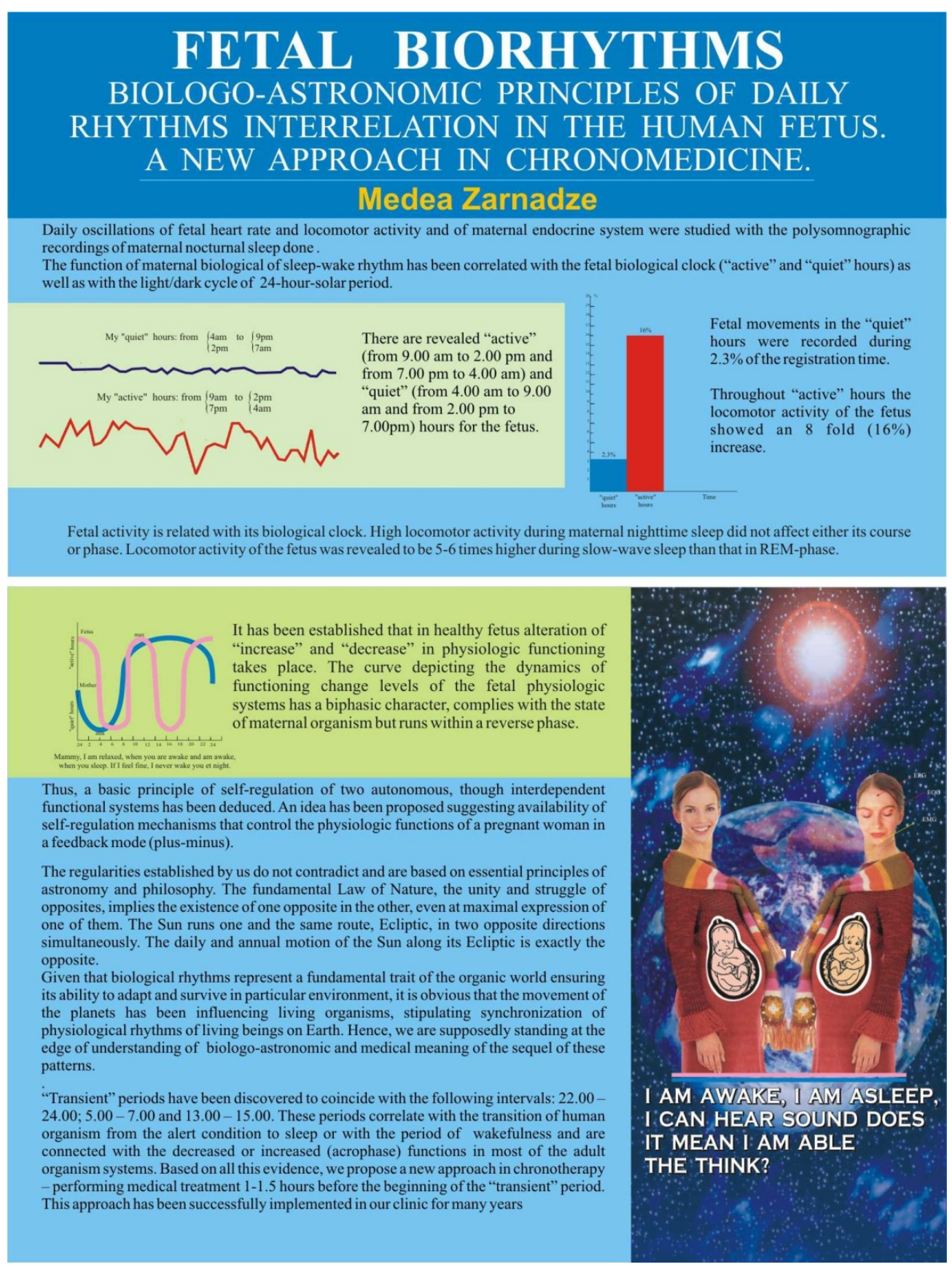




\section{Submit or recommend next manuscript to SCIRP and we will provide best service for you:}

Accepting pre-submission inquiries through Email, Facebook, LinkedIn, Twitter, etc.

A wide selection of journals (inclusive of 9 subjects, more than 200 journals)

Providing 24-hour high-quality service

User-friendly online submission system

Fair and swift peer-review system

Efficient typesetting and proofreading procedure

Display of the result of downloads and visits, as well as the number of cited articles

Maximum dissemination of your research work

Submit your manuscript at: http://papersubmission.scirp.org/ 